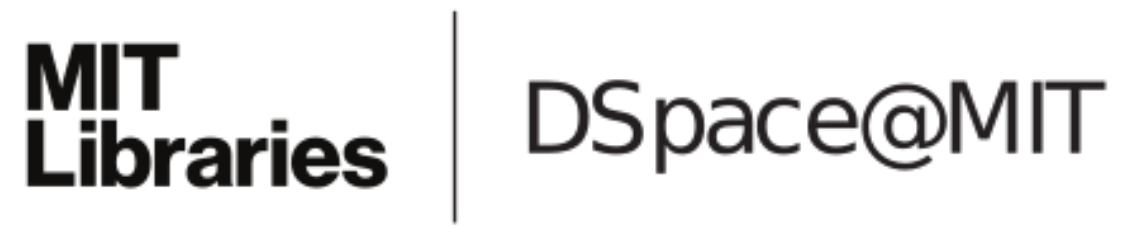

MIT Open Access Articles

Robust optimization: Lessons learned from aircraft routing

The MIT Faculty has made this article openly available. Please share how this access benefits you. Your story matters.

Citation: Marla, Lavanya, Vikrant Vaze, and Cynthia Barnhart. "Robust optimization: Lessons learned from aircraft routing." Computers \& Operations Research 98 (October 2018): pp. 165-84.

As Published: 10.1016/J.COR.2018.04.011

Publisher: Elsevier BV

Persistent URL: https://hdl.handle.net/1721.1/124319

Version: Author's final manuscript: final author's manuscript post peer review, without publisher's formatting or copy editing

Terms of use: Creative Commons Attribution-NonCommercial-NoDerivs License 


\title{
Robust Optimization: Lessons Learned from Aircraft Routing
}

\author{
Lavanya Marla ${ }^{\mathrm{a}, *}$, Vikrant Vaze ${ }^{\mathrm{b}}$, Cynthia Barnhart ${ }^{\mathrm{c}}$ \\ ${ }^{a}$ University of Illinois at Urbana-Champaign \\ ${ }^{b}$ Dartmouth College \\ ${ }^{c}$ Massachusetts Institute of Technology
}

\begin{abstract}
Building robust airline scheduling models involves constructing schedules and routes with reduced levels of flight delays as well as fewer passenger and crew disruptions. In this paper, we study different classes of models to achieve robust airline scheduling solutions, with a focus on the aircraft routing problem. In particular, we compare one domain-specific approach and two general paradigms of robust models, namely, (i) an extreme-value based or robust optimizationbased approach, and (ii) a chance-constrained or stochastic optimization-based approach. Our modeling and solution approach demonstrates the creation of data-driven uncertainty sets for aircraft routing using domain-specific knowledge and develops a completely data-driven simulation-based validation and testing approach. We first demonstrate that additional modeling, capturing domain knowledge, is required to adapt these general robust modeling paradigms to the aircraft routing problem, in order to meaningfully add robustness features specific to aircraft routing. However, we show that these models in their naive forms, still face issues of tractability and solution quality for the largescale networks which are representative of real-world airline scheduling problems. Therefore, we develop and present advanced models that address these shortcomings. Our advanced models can be applied to aircraft routing in multiple ways, through varied descriptions of the uncertainty sets; and moreover,
\end{abstract}

${ }^{*}$ Corresponding author

Preprint submitted to Computers and Operations Research

January 21, 2018 
are generally applicable to linear and binary integer programming problems. Through our detailed computational results, we compare the performance of solutions arising from these different robust modeling paradigms and discuss the underlying reasons for their performance differences from a data-driven perspective.

Keywords: robust airline scheduling, aircraft routing, robust optimization, chance-constrained programming

\section{Introduction}

Historically, airline schedule planners modeled the air transportation system assuming that flights can depart and arrive as scheduled. Factors such as weather, air traffic delays, mechanical problems and security issues, however,

5 regularly affect the Department of Transportation's (DoT) (US DoT, 2009) ontime performance metric, as evidenced by the 15-minute flight on-time arrival rate of $77.87 \%$ over the last 10 years (from Jan 2006 to Dec 2015) (Bureau of Transportation Statistics, 2016). In other words, over $22 \%$ of all flights during this period were either delayed upon arrival by at least 15 minutes $(20.14 \%)$, or cancelled $(1.75 \%)$ or diverted $(0.24 \%)$. Of the $20.14 \%$ flights delayed upon arrival by at least 15 minutes, more than a third $(7.12 \%)$ were due to aircraft arriving late from the previous flight in their itinerary (Bureau of Transportation Statistics, 2016). Similarly, several of the cancelled flights are likely to be so due to the aircraft arriving late from their previous flights, though such data 15 is not publicly reported. These delays and disruptions (like cancellations and diversions) are highly detrimental in an industry where the profit margins are typically small, with delay and fuel costs forming a major component of operating costs (Air Traffic Association, 2008). As a case in point, the total costs of U.S. domestic air traffic delays and disruptions in year 2007 were estimated at approximately $\$ 31.2$ billion (Ball et al. 2010 ). Of these, $\$ 8.3$ billion represents incremental operating costs due to delays to the airlines (including additional fuel, labor and material costs), $\$ 16.7$ billion represents the estimated costs to 
the passengers, and the remaining $\$ 6.2$ billion represents indirect costs in the form of lost demand and impact on GDP (Ball et al., 2010). Note that, while the delays have decreased slightly since they reached their highest levels in 2007, large delays are still prevalent. For example, across the last 10 years, the US flight cancellation rate in the year 2014 was the highest (even higher than that in 2007), while the on-time performance in 2014 was the worst since 2008. Thus, it is evident that delays and disruptions continue to be a major concern in the

30 US aviation system and if delays can be reduced, the airline industry and our society would benefit tremendously.

Robust airline scheduling is a way of pro-actively considering such delays and disruptions and creating schedules that are less susceptible to disturbances, or, easier to repair once disrupted. This is in contrast to prior practice, where the delay mitigation efforts are often reactive, that is, after an event occurs. These reactive efforts, called schedule recovery actions, can be costly and complex to implement. While schedule recovery may not be completely avoided even with robust solutions, the extent of recovery and its costs can be mitigated. Robust airline scheduling, then, is a proactive planning technique aimed at reducing total realized costs, including both planned and recovery costs.

We add a note here on the schedule recovery process at airlines. Usually recovery measures are undertaken when either a major weather event is anticipated in the near future, or the delay incurred till then exceeds a "large enough' value that requires intervention through recovery measures. The definition of 'large enough' depends on the operations management team's policies, and thus can differ significantly between airlines. Moreover, since recovery actions involve multiple resources, airlines may choose to undertake recovery either sequentially or simultaneously. For example, they could sequentially recover aircraft, followed by crew (which would be constrained by aircraft recovery decisions), followed by passengers. Alternatively, it is possible to integrate the decisions across the multiple resources which would have greater flexibility but also greater computational complexity. Moreover, these processes differ across airlines because different airlines may have somewhat different sets of priorities 
and may assess their available tradeoffs differently. Therefore, in this paper we focus on a version of robustness that makes schedules less susceptible to disruptions, rather than relying on explicitly evaluating the costs of recovery actions. We aim to generate robust schedules that are less likely to be inoperable as planned due to disruptions during the course of operations.

Ours is a data-driven study wherein we use simulation driven by empirical data to evaluate the true level of robustness of the obtained solutions, because the objective function values of the planning optimization models do not necessarily reflect the realized costs or the actual robustness level of the solution. Instead, we evaluate the solutions through a Monte-Carlo simulation using datadriven distributions and measure their performance with respect to a wide range of relevant robustness metrics.

\subsection{Motivation}

In this paper, our focus is on the aircraft routing step of the airline scheduling process. The aircraft routing problem is to find a feasible sequence of flight legs, called an aircraft routing or rotation, to be operated by each aircraft so that maintenance restrictions on aircraft are satisfied. Each flight is required to be assigned to (or covered by) exactly one aircraft, using no more than the available number of aircraft and meeting all maintenance requirements. Though robust planning adds value at every step of the airline scheduling process, we choose aircraft routing because of its high impact on schedule reliability and relatively

75 low impact on flight operating costs, crew costs, and passenger revenues (Lan et al. 2006). We define robust airline schedules as those schedules that are less likely to be inoperable as planned due to disruptions occurring during the course of operations.

Let us demonstrate how aircraft routings differ in robustness and what we mean by robust aircraft routings, with an example. In Table 1, we report performance of seven aircraft routing solutions as measured by the percentage of flights in the routing solution that arrive within 15 minutes, 30 minutes, 60 minutes, 120 minutes and 180 minutes of their respective scheduled arrival times. 
Note that these percentages, for this illustrative example, were calculated over 22 selected days of operations of a major U.S. airline. For the instances under consideration in this paper, all of which are drawn from actual airline operations, we compare various metrics of interest, as detailed in $\$ 3$. The reported variability in flight delays is significant, as even small differences of around $1 \%$ can increase/decrease the airline's ranking with respect to the DoT's 15-minute on-time performance metric (Bureau of Transportation Statistics, 2009b). If the airline does not explicitly consider delays in selecting aircraft routings, the airline may end up choosing any of these routings, and thus, can potentially incur high delays. To illustrate, for this instance, the aircraft routing actually operated by the airline is Routing Solution 5. Incidentally, the DoT on-time 95 performance ranking for this airline during this time period was third from the bottom. Moreover, in addition to aircraft delay disparities, different routings can lead to different levels of passenger disruptions and delays. A passenger is considered to be disrupted if one or more flight legs in his/her itinerary are canceled, or if delays leave insufficient connection time to the next flight leg in his/her itinerary causing a misconnection. The second from last column of Table 1 shows the number of disrupted passengers for each routing solution and the last column shows the percentage reduction in the number of disrupted passengers when compared with the airlines actual routing solution (Routing Solution 5). Routing Solutions 1 and 2 can vastly improve upon the airline's solution on each of these metrics without requiring any additional resources, while Routing Solution 7 can further deteriorate the airline's performance on these metrics greatly. Therefore, it is clear that considering robustness explicitly is important.

\subsection{Propagated Delays and Robustness Metrics}

The relationship between aircraft routings and delays and disruptions can be explained by the phenomenon of propagated delays. In terms of causes, flight delays can be divided into two components (Lan et al., 2006): independent delays that originate at the flight's origin or during the flight, and propagated 


\begin{tabular}{c|ccccc|cc}
\hline & \multicolumn{4}{|c|}{ \% Flights with Lower or Equal Delays } & \multicolumn{3}{c}{ Pax Disruptions } \\
\hline Routing Solution & $\leq 15 \mathrm{~min}$ & $\leq 30 \mathrm{~min}$ & $\leq 60 \mathrm{~min}$ & $\leq 120 \mathrm{~min}$ & $\leq 180 \mathrm{~min}$ & \#D-pax & \% Reduction in D-pax \\
\hline Routing Solution 1 & 79.1 & 86.7 & 93.4 & 98.0 & 99.1 & 988 & 10.1 \\
Routing Solution 2 & 78.8 & 86.8 & 93.2 & 98.2 & 99.2 & 986 & 10.3 \\
Routing Solution 3 & 78.3 & 86.2 & 92.9 & 98.1 & 99.0 & 1028 & 6.5 \\
Routing Solution 4 & 78.3 & 86.0 & 92.9 & 97.5 & 98.6 & 1047 & 4.8 \\
Routing Solution 5 & 77.7 & 85.8 & 92.8 & 97.7 & 98.9 & 1100 & 0 \\
Routing Solution 6 & 77.6 & 85.7 & 92.4 & 97.4 & 98.6 & 1057 & 3.9 \\
Routing Solution 7 & 76.5 & 84.7 & 92.0 & 97.2 & 98.5 & 1223 & -11.2 \\
\hline
\end{tabular}

Table 1: Flight Delay Distributions and Passenger Disruptions for Different Routing Solutions

delays resulting from delays in upstream flights that are not absorbed by slack

time between flight legs. Note that the term independent delays is meant to convey the fact that these delays are assumed to be independent of the aircraft routings being used and are not assumed to be (statistically) independent of each other. In fact, as will be seen in our computational experiments, we do consider situations where the independent flights delays are both correlated and uncorrelated with each other. Sometimes, these independent delays are also called as the root delays or as primary delays. However, we will use the phrase independent delays to remain consistent with the terminology used by Lan et al. (2006).

Delay propagation is illustrated in Fig. 1. The solid arrows show the planned schedule for flights $f_{1}$ and $f_{2}$; and the dotted arrows the operated schedule. $P D T, A D T, P A T$ and $A A T$ are the planned departure time, actual departure time, planned arrival time and actual arrival time respectively, of flight $f_{2}$. In Figure 1. flight $f_{1}$ is delayed, and its delay causes the remaining time between flight $f_{1}$ 's actual arrival and $f_{2}$ 's scheduled departure to be less than the minimum connection time required for the same aircraft to fly both flight legs. This causes propagated delay $P D$ for flight $\operatorname{leg} f_{2}$. In addition, independent delay is incurred by $f_{2}$, both at its departure $(I D D)$ and its arrival $(I A D)$, resulting in total departure delay $(T D D)$ and total arrival delay $(T A D)$. However, by changing the sequence of flights operated by each aircraft, propagated delay can be reduced (Figure 2). This involves changes in the routings of aircraft. 


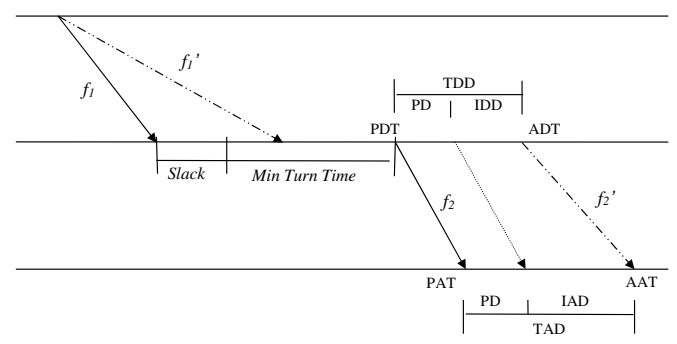

Figure 1: Delay Propagation along an Aircraft Route

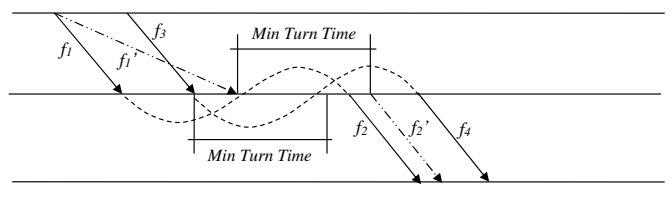

Original routing

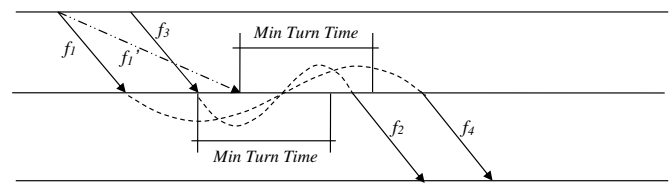

New robust routing

Figure 2: Robust Routing with Optimal Slack Allocation

Because no new aircraft are being added and the flight schedule remains unchanged, the total slack in the system is not altered, instead only the positioning of the slack is changed. Also note that through this change in routing, we are able to influence the propagated delay and total delay, while independent delays, by definition remain unchanged. Thus, for a given routing of aircraft, the total propagated delay will differ from the total delay by a constant value equal to the total independent delay.

For the airline studied in this paper, propagated delay typically represents $20 \%$ to $30 \%$ of total flight delay. Because total independent delay is a constant for the flight schedule, reducing delay propagation by choosing Routing Solution 1 instead of, for example, Routing Solution 7 (see Table 1), has the effect of 


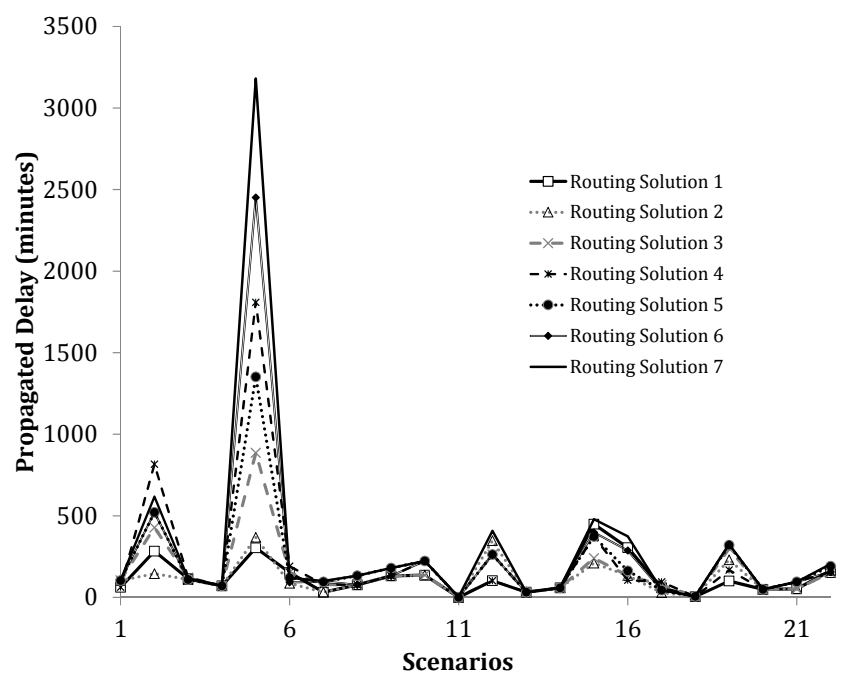

Figure 3: Propagated Delays of Feasible Aircraft Routings, $N_{2}$

reducing total flight delay. The differences in propagated delays for Routing Solutions 1-7 are shown in Fig 3. Each scenario here represents a different possible combination of independent delays. Different aircraft routing solutions are not very different for 'good' scenarios like Scenario 10, but differences become apparent for 'bad' scenarios like Scenario 5.

Metrics of Robustness: Good solutions to airline scheduling problems will have lower levels of flight delays, and good travel experience due to lower passenger delays and fewer passenger disruptions. Metrics such as total flight delay minutes, total cost of delay, 15-minute on-time performance, 30-min on-time performance, and 60-minute on-time performance, are all examples of measures that reflect airline schedule reliability and robustness. One problem, however, is that these metrics are not always aligned with each other. For example, the 15-minute on-time performance metric does not get affected by delays greater than 15 minutes, and therefore maximizing 15-minute on-time performance is not the same as minimizing total delay minutes. Similarly, minimizing aircraft delay minutes is different from minimizing passenger delay minutes or minimizing the number of passenger disruptions. In this paper, we will evaluate 
robustness via aircraft delay-based metrics using different delay thresholds as

\subsection{Existing Work}

Literature on building robust solutions to optimization problems falls into three major categories: domain-specific approaches, probability distributionbased approaches, and probability distribution-free approaches. These different approaches attempt to capture uncertainty in differing ways.

\subsubsection{Domain-Specific Approaches to Robustness}

There is a rich set of literature on domain-specific approaches to robustness in airline schedule planning. These approaches identify specific features of the problem of interest that affect solution robustness. Eggenberg et al. (2008) discuss a number of these features. In this section, we only present a summary of some of the representative studies in this area. But, we discuss in more detail the models that involve robust aircraft routing.

Ehrgott and Ryan (2002) provide a bicriteria optimization between planned cost and robustness, seeking to maximize robustness within allowable budgets of deviation from the minimum cost solution. Robustness is defined in terms of having the feature of sufficient ground times if crews have to change aircraft, or as keeping the crew with the aircraft in case of tight ground times. Ageeva (2000) creates robust aircraft routing solutions by building routings that contain more 'swap' opportunities. The author examines multiple optimal solutions to the aircraft routing problem and ranks them by their robustness, as measured by the number of swaps. However, these two studies do not evaluate the resulting robust solutions through simulation. Kang and Clarke (2002) present an approach to improve operational robustness using degradable airline scheduling. Prioritizing on the basis of revenue, schedules are divided into layers that mini190 mize cross-layer interactions, so that any recovery actions taken in one layer will not influence higher priority layers. Different models of incorporating degradability are presented, including degradable schedule partitioning, degradable 
fleet assignment, and degradable aircraft routing. Rosenberger et al. (2004) develop a robust model for fleet assignment and aircraft routing that maximizes the feature of 'short cycles'. Under disruptions, when recovery is undertaken, airlines cancel not just one flight, but typically a cycle containing that flight, so as to reposition the aircraft to operate the rest of the schedule as planned. By increasing the number of short cycles, i.e., cycles containing a small number of flights, they can decrease the number of flights canceled. Evaluating the solutions via simulation, the authors show that the incorporation of robustness in the form of short cycles produces fleet assignment solutions that decrease planned operating costs and passenger spill. Smith and Johnson (2006) solve the fleet assignment problem by limiting the number of aircraft types that are allowed to operate in each airport, and thus limit aircraft dispersion. They show that this measure can help other aspects of airline schedule planning, such as maintenance routing, crew scheduling, etc., in making operations more robust.

Schaefer et al. (2005) introduce a measure to evaluate crew schedules, by incorporating planning costs and approximating the operational costs of crew schedules. They propose a combination of two methods: one based on expected operating cost, in which disruptions are generated using SimAir, a Monte-Carlo simulation of airline operations; and another based on penalizing pairing features that may result in poor delay performance. Using simulation, they illustrate that solutions generated using these approaches perform better under uncertain conditions compared to deterministic approaches that do not take uncertainty into account. Yen and Birge (2006) model crew scheduling as a two-stage stochastic program and show tradeoffs between planned crew costs and recourse costs. The first stage of the model minimizes expected crew costs. The second stage recourse model minimizes the expected costs of crews swapping between planes under scenarios of uncertainty. Shebalov and Klabjan (2004) propose the concept of move-up crews and improve costs by swapping crews, and show the resulting benefits.

Jiang and Barnhart (2009) address demand uncertainty by introducing fleet re-timing as a dynamic scheduling mechanism and supplement re-fleeting with 
re-timing. These features affect the number of connecting itineraries available legs within a small time window without significantly increasing costs. Their approach is very relevant to our work, and will be discussed in greater detail in later sections. AhmadBeygi et al. (2010) expand on this notion of propagated 


\subsubsection{General Paradigms of Robust Models}

Distribution-free and distribution-based approaches capture parameter uncertainty in a variety of ways. These approaches rely on two types of user inputs, namely, uncertainty sets and protection parameters. Uncertainty sets 


\begin{tabular}{|c|c|c|c|c|}
\hline & Schedule Design & Fleet Assignment & Aircraft Routing & Crew Scheduling \\
\hline Ageeva (2000) & & & $\bullet$ & \\
\hline Ehrgott and Ryan 2002. & & & & $\bullet$ \\
\hline Rosenberger et al. (2004) & & $\bullet$ & $\bullet$ & \\
\hline Kang and Clarke 2002 & $\bullet$ & $\bullet$ & $\bullet$ & \\
\hline Shebalov and Klabjan (2004. & & & & $\bullet$ \\
\hline Schaefer et al. (2005) & & & & $\bullet$ \\
\hline Lan et al. (2006) & $\bullet$ & & $\bullet$ & \\
\hline Smith and Johnson (2006) & & • & & \\
\hline Yen and Birge 2006$)$ & & & & $\bullet$ \\
\hline \begin{tabular}{|l|l|} 
Jiang and Barnhart & 2009. \\
\end{tabular} & • & • & & \\
\hline AhmadBeygi et al. (2010) & & & $\bullet$ & \\
\hline Weide et al. 2010 & & & - & $\bullet$ \\
\hline Burke et al. 2010 & $\bullet$ & & - & \\
\hline Sohoni et al. 2011) & $\bullet$ & & & \\
\hline Dunbar et al. 2012. & & & $\bullet$ & \\
\hline Eggenberg et al. (2011). & $\bullet$ & & & \\
\hline Sohoni et al. (2011) & $\bullet$ & & & \\
\hline Arikan et al. 2013. & $\bullet$ & & & \\
\hline Froyland et al. (2014b) & & & $\bullet$ & \\
\hline Froyland et al. 2014a) & & & $\bullet$ & \\
\hline Yan and Kung 2015. & & & $\bullet$ & \\
\hline
\end{tabular}

Table 2: Summary of Domain-Specific Literature 

anism to control the degree of conservatism through protection parameters $\Gamma$, which specify the number of uncertain parameters that can simultaneously take on their worst-case values. For integer values of $\Gamma_{i}$, the formulation protects 
against the case when $\Gamma_{i}$ parameters in constraint $i$ assume their worst-case „values and all other parameters assume their nominal values. Bertsimas and Sim (2004) use linear uncertainty sets in which each parameter varies in a fixed range around its nominal value. Other approaches have defined uncertainty sets differently; for example, some have defined ellipsoidal uncertainty sets. We refer the reader to Ben-Tal and Nemirovski $(1999,2000,1998)$ for further details on this topic.

\subsubsection{Chance-Constrained (Distribution-Based) Paradigm}

Distribution-based approaches are those that assume knowledge of a probability distribution for the uncertain parameters. Chance-constrained programming (CCP), specifically, admits random data variations and permits constraint violations up to certain specified probability limits (Charnes and Cooper, 1963). Unlike in stochastic programming the decisions are not required to be feasible for every realization of the random parameters (as defined by the uncertainty set), but feasibility is ensured with at least some specified probability. The uncertainty set in this case is defined by the probability distribution or by a set of scenarios that is representative of the probability distribution. When uncertainty in a constraint of a linear program is limited to the right-hand-side, Charnes and Cooper (1963) show that the randomness component can be modeled deterministically and the model can be converted into a linear program with very little increase in complexity. The model is intuitively appealing, but requires at least partial knowledge of data distributions, compared to a distribution-free approach. The uncertainty set can then be described in terms of information about quantiles of the uncertain parameters (Marla, 2007). CCP models, however, face computational issues under uncertainty in the left-hand-side of the constraint matrix, because the usage of multinomial distributions becomes necessary. This difficulty may be surmounted by describing the uncertainty set in terms of representative scenarios, either sampled from a distribution or obtained from historical data, which gives computationally tractable approximations of the CCP formulations (Nemirovski and Shapiro, 2006). 


\subsection{Contributions}

Our work focuses on a fully data-driven approach to studying the three paradigms in the context of the robust aircraft routing problem. We study the performance of a domain-specific approach (Lan et al., 2006), and two general paradigms - the Extreme-Value(EV)-based distribution-free robust optimization approach of Bertsimas and Sim (Bertsimas and Sim, 2004, 2003) and the probability distribution-based Chance-Constrained Programming approach (CCP) (Charnes and Cooper, 1959, 1963). A delay simulator is embedded within the modeling framework to parameterize the robust formulations. Additionally, the validation and testing of the solutions from the models is done via a data-driven, simulation-based evaluator.

We focus on the most straightforward implementations of the extreme-valuebased and chance-constrained paradigms to aircraft routing. While several other implementations under these same paradigms could exist, (especially for special cases such as pre-specified and correlated delay uncertainty sets, see Yan and

${ }_{60}$ Kung (2015)), our goal is to understand the functioning of these general robust paradigms relative to each other and relative to domain-specific approaches. Therefore, we focus on comparing these general models without biasing them in favor of specific cases.

Our first contribution is to demonstrate how uncertainty is modeled in composite variable-based formulations through the EV and CCP paradigms, and then show how to model the robust aircraft routing problem using the aforementioned three modeling approaches. Second, we demonstrate that existing protection-level based models, like CCP and EV, suffer from issues of non-monotonicity due to lack of pareto-optimality of their solutions. Third, we develop new models in the extreme-value and chance-constrained modeling paradigms aimed at addressing the identified limitations related to monotonicity, ease of modeling and tractability. Fourth, we examine and compare the solution quality of the robust solutions from these different modeling approaches and provide insights in terms of the differences in solution quality, ease of implemen-

tation and tractability. Note that the findings and extensions from this work 
are generally applicable to the broad class of network-based resource allocation problems (Marla, 2007).

\subsubsection{Outline}

In Section 2, we present the three classes of robust models studied in this paper. We present the basic aircraft routing model, and discuss the method to capture uncertainty in the composite variable setting. We then present Charnes and Cooper's Chance-Constrained Programming approach, Bertsimas and Sim's robust optimization approach, and Lan, Clarke and Barnhart's robust aircraft routing approach. In Section 3 we present the experimental set-up for our computations, and in Section 4 we study the performance of the different models using the simulator presented in Section 3.4 . In Section 5 we propose extensions and enhancements to these general classes of models that address the limitations of the models described in Section 2. We compare the performance of these enhanced robust aircraft routing models in Section 6, in terms of their complexity and run times (Section 6.1), model parameters (Section 6.2) and modeling paradigms (Section 6.3), after evaluating the performance of these solutions via simulation. We conclude with insights into the differences between modeling paradigms and their relative performances. In Section 7, we summarize our findings.

\section{Robust Models}

\subsection{Deterministic Aircraft Routing Model}

Following is the standard deterministic aircraft routing formulation, denoted by $A R$, which we attempt to make robust in the subsequent sections. We use this model as a benchmark model to describe the remaining models in the paper. 
AR :

$\min 0$

$$
\begin{array}{lr}
\text { s.t. } \sum_{s \in S} a_{i s} x_{s}=1 & \forall i \in F \\
\sum_{s \in S_{i}^{+}} x_{s}-y_{i, d}^{-}+y_{i, d}^{+}=0 & \forall i \in F^{+} \\
-\sum_{s \in S_{i}^{-}} x_{s}-y_{i, a}^{-}+y_{i, a}^{+}=0 & \forall i \in F^{-} \\
\sum_{s \in S} r_{s} x_{s}+\sum_{g \in G} p_{g} y_{g} \leq N & \\
y_{g} \geq 0 & \forall g \in G \\
x_{s} \in\{0,1\} & \forall s \in S
\end{array}
$$

The decision variables in this formulation correspond to strings (sequences of flight legs) with each string starting and ending at a maintenance station and obeying FAA (Federal Aviation Administration) and other regulations regarding maximum time between maintenance checks. Strings capture multiple decisions simultaneously, and thus are what we refer to as composite variables Armacost 405 et al. 2002). These are typically used to yield strong formulations, and/or when they eliminate the need for complex, difficult to model, constraints, as in the case of aircraft routing.

We set up a time-space network representation of the network (Marla et al. 2016). A maintenance station is any airport where aircraft maintenance can be performed. Let $F$ be the set of daily flight legs, $F^{+}$be the set of flight legs originating at a maintenance station, $F^{-}$be the set of flight legs ending at a maintenance station, and $S$ be the set of possible strings. The set of ground arcs (including wraparound arcs which begin on one day and end on another day at the same location) is denoted by $G$, the set of strings ending with flight ${ }_{415} \operatorname{leg} i$ is $S_{i}^{-}$, and the set of strings beginning with flight leg $i$ is $S_{i}^{+}$. Ground variable $y_{i, d}^{-}$represents the number of aircraft on the ground before flight leg $i$ 
departs and $y_{i, d}^{+}$is the number of aircraft on the ground after flight leg $i$ departs. Similarly $y_{i, a}^{-}$is the number of aircraft on the ground before flight leg $i$ arrives and $y_{i, a}^{+}$is the number of aircraft on the ground after flight leg $i$ arrives. $a_{i s}$ is 1 if flight leg $i \in F$ is contained in string $s \in S$ and 0 otherwise. $r_{s}$ is the number of times string $s \in S$ crosses the count line, $p_{g}$ is the number of times ground arc $g \in G$ crosses the count line, and $N$ is the number of aircraft, of the fleet type under consideration, available to the airline. Constraints 2 are the cover constraints that require each flight leg to be covered by some aircraft exactly once. Constraints (3) and (4) balance the number of aircraft at each airport location, and constraints (5) count the number of aircraft. Decision variable $x_{s}$ takes on value 1 if string $s \in S$ is selected to be operated by an aircraft, and 0 otherwise. Decision variable $y_{g}$ is the number of aircraft on ground arc $g \in G$.

\subsection{Modeling Uncertainty in Composite Variable Coefficients}

The first set of challenges associated with achieving robust solutions are related to deciding what constitutes robustness and how to capture that in the optimization model. The sources of uncertainty, as discussed in Section 1.2 are independent delays of flights. However, the model formulation (1) - (7) has string composite variables and associated coefficients. Therefore, we need to be able to relate how uncertainty that is rooted at the flight-level translates to uncertainty at the string-level, and then capture that as uncertainty in the parameters of the optimization formulation (1) - (7). We do this by means of a data-driven simulator described below.

\subsubsection{Simulator}

440 Using schedule data and details of operated aircraft routings (available from the AOTP database (Bureau of Transportation Statistics, 2009a)), we compute the independent and propagated delay for each flight leg, in each of the simulation scenarios, using a method similar to the method used in Lan, Clarke and Barnhart (Lan et al., 2006). This is possible because independent delays are, by definition, independent of aircraft routings. The propagated delays in each 
scenario are computed as discussed in Section 1.2 . Thus, by applying independent delays to any sequence of flights forming a string we estimate, for each scenario and for each flight leg $i$ along that string, propagated delay $\left(P D_{s}\right)$, total departure delay $\left(T D D_{i}\right)$ and total arrival delay $\left(T A D_{i}\right)$. With this simulator, it is also possible to compute the probability of a flight leg being delayed by at least a specified threshold, or the probability of a string experiencing at least a specified level of propagated delay, or the range of delays experienced by a flight leg or a string. These various characterizations of uncertainty are employed to compute the robust optimization model inputs. Please note that what we call as a simulator in this section does not conduct any Monte-Carlo or probabilistic simulations. It simply implements the algorithm proposed by Lan et al. (2006) to calculate the propagated delays for all strings under several scenarios in a deterministic manner. It is called a simulator because it helps simulate the propagated delays for even those strings which were never operated in the historical schedules, thus making them available for being chosen in a routing solution.

Next, we illustrate different modeling paradigms along with the associated computational challenges and their solutions.

\subsection{Domain-Specific Approach}

Lan, Clarke and Barnhart (Lan et al., 2006) describe an approach to develop robust aircraft routings by modifying the benchmark model $A R$ to minimize the total expected string propagated delay. The benchmark model is modified to add an objective function that minimizes the expected value of the sum of the propagated delays across all strings in the solution. Let $p d_{i j}^{s}$ be the propagated delay from flight leg $i$ to flight leg $j$ when $i$ immediately precedes $j$ in string $s$. This can be computed using the simulator (Section 2.2.1) for each scenario in the historical data. Using the notation introduced in Section 2.1, the expected propagated delay in the network is: 


$$
E\left[\sum_{s \in S}\left(\sum_{(i, j) \in s} p d_{i j}^{s}\right) x_{s}\right]=\sum_{s \in S}\left(x_{s} \times \sum_{(i, j) \in s} E\left[p d_{i j}^{s}\right]\right)=\sum_{s \in S} d_{s} x_{s}
$$

where $d_{s}=\sum_{(i, j) \in s} E\left[p d_{i j}^{s}\right]$.

Recall from Section 1.1 that propagated delay differs from total flight delay by a constant value. Thus, minimizing propagated delay is equivalent to minimizing total flight delay.

In terms of problem structure and complexity, the domain-specific model by Lan, Clarke and Barnhart $(L C B)$ is the same as $A R$, except that the feasibility objective is replaced with the objective of minimizing expected propagated delay, specifically:

\section{LCB :}

$\min \sum_{s \in S} d_{s} x_{s}$

s.t. Cover, Balance, Count, Non-negativity, and Integrality (2) - 7) (10)

\subsection{Probability Distribution-Based Chance-Constrained Programming Approach}

In Chance-Constrained Programming, the chance or probability that a constraint of the model is satisfied is required to be at least equal to some specified threshold probability level. One way to apply it to the original formulation of the aircraft routing problem (1) - (7), is by modifying the cover constraint (2). Relative to the benchmark model that has a cover constraint that ensures each flight is covered in exactly one string the solution, the chance-constrained version of (2) specifies that the probability that each flight is covered is at least equal to a certain user-specified probability. The chance-constrained aircraft routing formulation is as follows: 
$\max 0$

$$
\text { s.t. } \mathbf{P}\left(\sum_{s \in S} a_{i s} x_{s}=1\right) \geq \alpha_{i}
$$

$\forall i \in F$

Balance, Count, Non-negativity, and Integrality $(3)-(7)$,

where $\mathbf{P}$ denotes probability, and $\alpha_{i}$ is a user-defined protection parameter indicating the minimum probability that the aircraft routing solution will satisfy constraint (2) for flight $i$. Charnes and Cooper (1959) describe how to model the non-linear constraints $(12)$ as linear constraints.

\subsubsection{Uncertainty Set Construction}

For this formulation, as discussed in Section 1.3.2, we are required to define the uncertainty sets and the protection parameters. An uncertainty set specifies the set of realizations of parameters that the formulation may protect against.

In chance-constrained programming, usually, probabilistic descriptions or their scenario-based approximations are used as uncertainty sets. In this paper, we utilize historical data to obtain empirical distribution information, and that combined with domain knowledge of airline industry helps us define the uncertainty sets. Because it is not possible to cover a flight (without delays) $100 \%$ of the time, the protection level $\alpha$ specifies the frequency with which a given level of delays are protected against.

Our uncertainty set is constructed in a data-driven manner. From historical delay scenarios, we group the delay incurred by flight $i$ in string $s$, into intervals of delays in minutes: $[0,15],(15,30],(30,60],(60,90],(90,120],(120,180]$ and $(180, \infty)$. We define a flight as being covered, if it can be operated by a string $s$ without a delay greater than a threshold delay $t$. For example, at delay levels of $t=15$ minutes (beyond the $[0,15]$ minute interval), flights incur delays which are directly related to the DoTs 15 -minute on-time performance metric; at $t=$ 30 minutes (beyond the $[0,30]$ minute interval), connecting passengers typically risk missing their connecting flight; and at $t=90$ minutes (beyond the $[0,90]$ 
minute interval), flight cancellations may occur as airlines are likely to cancel flights that are expected to be over 90 minutes late. Note that, the thresholds for misconnections and cancelations are not exact, but are good approximations based on, respectively, typical passenger connection times and typical airline operational strategies. Thus our uncertainty set is defined in a data-driven manner which is informed by our domain knowledge. Specifically, empirical distributions are derived from historical data and domain knowledge related to flights' delays, passengers' misconnections and flight cancelations informs the choice of $t$. Our uncertainty set, therefore, is parameterized by $t$. The scenarios in the uncertainty set of interest have different interpretations depending on the choice of $t$.

\subsubsection{Protection Parameters and Formulation}

We model uncertainty probabilistically in the flight cover constraints 2 by defining $p_{i s}$ as the probability obtained from historical data, that flight leg $i$ in string $s$ is covered according to the required service level, that is, its arrival is delayed by less than or equal to $t$ minutes when string $s$ is operated. We obtain $p_{i s}$ from the simulator, for all possible flight-string combinations, over all scenarios.

Because each flight leg is present only in a single string in the solution, the 535 probability of flight $i$ being delayed less than or equal to $t$ minutes is $p_{i}=$ $\sum_{s \in S} p_{i s} x_{s}$. Using this, we re-write 111 - 13) as:

\section{$\mathrm{CCP}_{\mathrm{f}}$ :}

$$
\max 0
$$

$$
\begin{array}{ll}
\text { s.t. } \sum_{s \in S} a_{i s} x_{s}=1 & \forall i \in F \\
\sum_{s \in S} p_{i s} x_{s} \geq \alpha_{i} & \forall i \in F
\end{array}
$$

Balance, Count, Non-negativity, and Integrality (3) - 7) 
Constraints 16 are the robustness constraints that limit to $1-\alpha_{i}$ the probability that flight leg $i$ is delayed more than $t$ minutes. In structure and complexity, $C C P_{f}$ is similar to $A R$, though it adds constraints (16), one for each flight leg, and requires specification of the value of $\alpha_{i}$ for each constraint $i \in F$. A challenge associated with this model is the specification of $\alpha_{i}$ values. Values that are too high can lead to infeasibilities and values that are too low can lead to inadequate levels of robustness or protection. As a result, $C C P_{f}$ might have to be solved repeatedly to find appropriate $\alpha$-values by trial-and-error. Repeated solution of $C C P_{f}$, however, might be impractical in identifying the best set of $\alpha$ values.

\subsection{Extreme-Value Robust Optimization Approach}

Relative to the $A R$ model, the $E V$ model based on the Bertsimas and Sim robust optimization approach ensures that each flight is covered in the case when a subset of flights take on their worst-case delay values. We provide further details below. For applying the extreme-value (EV) robust optimization approach of Bertsimas and Sim to the aircraft routing problem, we define the uncertainty set in a manner similar to that described in Section 2.4. $\tilde{a}_{i s}$ are the realizations of $a_{i s}$ parameters under uncertainty, with $a_{i s}$ itself being the nominal value without uncertainty. Under this approach, $\hat{a}_{i s}$ is the width of the interval of uncertainty from the nominal, such that $a_{i s}+\hat{a}_{i s}$ is the worst-case realization of $\tilde{a}_{i s}$. We set $\hat{a}_{i s}=-1$ if flight $i \in F$ in string $s \in S$ has extreme value of delay exceeding $t$ minutes in the historical data. Because the Bertsimas and Sim approach considers realizations of the uncertain parameters at their extreme (or worst-case) values (Bertsimas and Sim (2004, 2003)), if a flight $i$ in string $s$ has extreme delay exceeding $t$ minutes using historical data, the extreme value $\hat{a}_{i s}=-1$ results in $a_{i s}+\hat{a}_{i s}=1-1=0$, reflecting the extreme occurrence that flight $i$ is not covered according to the required service level. Similar to the CCP model, $t$ can take on different values to define different uncertainty sets and associated worst-case scenarios. For example, as before, $t=15 \mathrm{~min}, t=$ $30 \mathrm{~min}$ and $t=90 \mathrm{~min}$, could represent on-time performance, missed passenger 
connections and flight cancellations respectively.

For each flight $i$, the Bertsimas and Sim robust optimization approach defines a 'robustness' or 'protection' parameter $\Gamma_{i}$ taking on (possibly continuous) 570 values in $[0,|S|]$, representing the number of coefficients in constraint $i$ that can assume worst-case or extreme values and still satisfy feasibility of the constraint. The model ensures constraint feasibility in the case where up to $\left\lfloor\Gamma_{i}\right\rfloor$ coefficients take on extreme values, and one coefficient $a_{i t}$ changes by up to $\left(\Gamma_{i}-\left\lfloor\Gamma_{i}\right\rfloor\right) \hat{a}_{i t}$. For our purposes, integer values of $\Gamma_{i}$ are the most meaningful because of the uncertainty definition. For each flight $i \in F, \Gamma_{i}$ represents the number of strings containing flight $i$, in which flight $i$ does not experience worst-case delays greater than or equal to $t$ minutes. The resulting extreme value formulation from the Bertsimas and Sim model is:

$\min 0$

$$
\begin{aligned}
\text { s.t. } & \sum_{s \in S} a_{i s} x_{s}=1 \\
& \sum_{s \in S} a_{i s} x_{s} \\
& +\max _{\left\{S_{i}^{\prime} \cup t_{i}\left|S_{i}^{\prime} \subseteq S,\right| S_{i}^{\prime} \mid=\left\lfloor\Gamma_{i}\right\rfloor, t_{i} \in S \backslash S_{i}^{\prime}\right\}}\left\{\sum_{s \in S_{i}^{\prime}} \hat{a}_{i s} w_{s}+\left(\Gamma_{i}-\left\lfloor\Gamma_{i}\right\rfloor\right) \hat{a}_{i t_{i}} w_{t}\right\}=1 \quad \forall i \in F
\end{aligned}
$$

$$
\begin{aligned}
& x_{s} \leq w_{s} \quad \forall s \in S \\
& w_{s} \geq 0 \quad \forall s \in S
\end{aligned}
$$

Balance, Count, Non-negativity, and Integrality (3) - (7)

If $\Gamma_{i} \geq 1$, the constraints 19$)$ and $(20)$ ensure that each flight $i \in F$ is covered by exactly one string $s$ which contains flight $i$ and has extreme value of delay less than $t$ for flight $i$. Because the values of $\hat{a}_{i s}$ are either 0 or -1 , (18)-23) simplifies to: 


\section{$\mathbf{E V}_{\mathbf{f}}$ :}

$\min 0$

s.t. $\sum_{s \in S} a_{i s} x_{s}+\max \left\{\sum_{s \in S} \hat{a}_{i s} x_{s},-\Gamma_{i}\right\}=1$

Balance, Count, Non-negativity, and Integrality $(3)-77$,

with the second term in the left-hand side of 25) called the protection function, which incorporates the protection level parameters $\Gamma_{i}$. This formulation

585 can be linearized easily as described in (Bertsimas and Sim, 2004).

Note that the robustness of the solution can also be varied by selecting the uncertainty set differently, through different values of the extreme delay parameter $t$. Reducing the value of $t$ will have the effect of generating more conservative solutions, those that permit little delay, while increasing the value of $t$ will have the opposite effect. In this research, we ran computational experiments using various different values for the the uncertainty set, as parameterized by different $t$ values. Our results and conclusions are based on these different experiments.

An alternative formulation of the extreme-value approach can be achieved by capturing uncertainty via the $d_{s}$ coefficients in the $L C B$ formulation, allowing the uncertainty to be captured at the string level (instead of the flight-string combinations, as with $E V_{f}$ ). This requires a completely different definition and parameterization of the uncertainty set, and the protection parameters. This formulation, denoted by $E V_{s}$, is presented in Appendix A and its applications to the aircraft routing problem are discussed in Section 5.2 .

\section{Experimental Set-up}

In this section, we introduce our experimental set-up and evaluation mechanism, through which we will demonstrate and evaluate the performance of our models. 


\subsection{Pre-Processing the Network Data}

which is publicly available as described in the previous paragraph. Because independent delays are assumed to stay constant regardless of the routing being used, we can later find the propagated delay for any routings, even the ones not 
used in the historical data.

After determining the independent delays of all flights that are operated on a daily basis (excluding weekends), we perform some further filtering by removing a handful of flights to ensure a balanced schedule, that is, the number of incoming flights is equal to the number of outgoing flights for each airport in

${ }_{640}$ the daily schedule. We select four such networks for our experiments, described in Section 3.2 .

Finally, for the selected networks, we generate a timeline network representation (Barnhart et al. 2003), with a time horizon of 72 hours. This horizon indicates that aircraft have repeating rotations that cover flights in a way that every 72 hours the aircraft can be serviced at a maintenance station at an airport. Under this representation, each aircraft string begins at a maintenance station and follows multiple consecutive flights with ground times in between. Each string ends with the last flight in it arriving at a maintenance station followed by some specified hours of ground time that allows for maintenance checks. We build strings using a depth-first-search algorithm that enumerates all strings in each of the selected networks. For the purposes of this study, we have chosen networks that have less than 1,000,000 strings.

\subsection{Description of Selected Networks}

We denote the networks by $N_{1}, N_{2}, N_{3}$ and $N_{4}$, each representing a different aircraft routing problem for a fleet type of an airline. Each of these networks in our dataset have daily repeating schedules, that is, each flight in these networks is operated every day by the exact same aircraft type. Various characteristics of $N_{1}, N_{2}, N_{3}$ and $N_{4}$ are shown in Table 3 . One subset of the independent delay data is used as input to the aircraft routing models to generate robust solutions under each modeling paradigm. These solutions are then evaluated using another subset of the data, corresponding to a future time period, to detect and avoid overfitting. 


\begin{tabular}{|c|c|c|c|c|c|}
\hline Network & Number of Daily Flights & Airports & Strings & Scenarios & Sampling \\
\hline$N_{1}$ & 50 & 16 & 9639 & 5000 & Distribution-fitting \\
\hline$N_{2}$ & 165 & 16 & 878207 & 200 & Direct AOTP data \\
\hline$N_{3}$ & 22 & 11 & 1959 & 102 & Direct AOTP data \\
\hline$N_{4}$ & 70 & 20 & 31800 & 120 & Direct AOTP data \\
\hline
\end{tabular}

Table 3: Fleet Network Characteristics

\subsection{Generating Scenario Data}

We filtered our dataset to ensure that we used data from only those days

where all the flights in our dataset were operated. In our dataset, each flight leg is operated several times, and we compute its independent delay value (as described earlier in Section 3.1 for each time the flight leg is operated in our filtered dataset. We regard each independent delay value (one for each day) as one scenario for this flight leg. In our data-driven approach, we assume that the independent delay of the flight legs has the same distribution as that given by the historical independent delay values. For networks $N_{2}, N_{3}$ and $N_{4}$, we have sufficient daily independent delay data in the year covered by our dataset. As shown in Table 3, there were 200, 102 and 120 repetitions respectively, of these flights available in our dataset. Therefore we use that data directly as scenarios in our experiments. In other words, for networks $N_{2}, N_{3}$ and $N_{4}$, each day in the historical data corresponds to a different delay scenario, which implies that any possible correlation between independent delays of different flight legs is automatically accounted for through the definition of our scenarios. For network $N_{1}$, we have about 60 scenarios over three months where all flights are operated. ${ }_{80}$ So, we fit a probability distribution to the historical independent delay values. After fitting multiple distribution families including gamma, lognormal, Gumbel and expoential distributions, lognormal distribution was found to have the best fit to the independent delay data. We then sampled from this distribution to generate 5000 scenarios as indicated in Table 3. We assume that the independent delays of different flights are statistically independent of each other for network $N_{1}$, though we are not required to make such an assumption for networks $N_{2}$, 
$N_{3}$ and $N_{4}$. Each of these two scenario generation approaches offers a different set of advantages and disadvantages; and together covers a wide spectrum of assumptions, thus leading to a more comprehensive evaluation of the models being considered in this paper.

\subsection{Metrics and Evaluator}

We compare the robustness of solutions from all the models in terms of three types of evaluation metrics: on-time performance, total propagated delay and passenger disruptions. For on-time performance, we look at 15-minute on- 
compute passenger disruptions, we enumerate all pairs of flights $\left(f_{1}, f_{2}\right)$ between which passengers connect. Let $C\left(f_{1}, f_{2}\right)$ be the scheduled time available for the passenger to make the connection and let $m_{f_{1} f_{2}}$ represent the minimum time needed for a passenger to connect between flights $f_{1}$ and $f_{2}$. If $C\left(f_{1}, f_{2}\right)-$ $T A D\left(f_{1}\right)+T D D\left(f_{2}\right) \leq m_{f_{1} f_{2}}$, the actual connection time between $f_{1}$ and $f_{2}$ is too short to connect and the passengers scheduled to make this connection are disrupted.

To ensure a fair comparison and also to avoid overfitting, we use a five-fold cross-validation approach. The data is divided into five subsets, and each time one of the five subsets is tested on the solution obtained from solving the model over the other four subsets. This is repeated with each subset iteratively as the test subset. We find and report the average performance of the model over all subsets. Our models are implemented in Java and CPLEX v12.51 on a Dell laptop with 16 GB RAM.

It is important to note that in evaluating robust routings, we assume that flight delays are allowed to propagate along the strings, without any recovery interventions such as cancelations or aircraft swaps. This allows us to estimate the levels of delay propagation and robustness of the strings without any intervention. Because cancelation and swapping strategies are different for different airlines and they depend on a variety of tradeoffs that have to be carefully made by the airlines, assuming particular swapping and cancelation strategies could lead to bias.

To avoid such bias, we compare different robust aircraft routing methods under the assumption that the airlines choose not to respond to large delays and disruptions, except in the most passive manner wherein the delays are allowed to propagate. On the one hand, this approach may be perceived as fair because it evaluates each robust aircraft routing method on its own merit, rather than having to rely on the assumption of a particular active recovery method that may modify its performance. On the other hand, this approach may be perceived as favoring the robust aircraft routing methods that rely less on active recovery actions. We acknowledge this second way of thinking. In fact, 
evaluating the combined effects of the various combinations of robust scheduling strategies and recovery strategies in a data-driven way would be the logical next step in this research. However, it is a substantially larger endeavor, and hence it is beyond the scope of this first paper. We identify that as an opportunity for future research.

\section{Preliminary Results and Motivation for Advanced Models}

The protection parameters $\alpha$ and $\Gamma$ in the $C C P_{f}, E V_{f}$ and $E V_{s}$ (described in detail in Appendix A models are designed to represent the desired extent of robustness, with larger values of $\alpha$ and $\Gamma$ representing higher levels of solution robustness. However, note that choosing a protection level $\alpha_{i}$ or $\Gamma_{i}$ for each constraint is a combinatorial decision, which is difficult given the large number of flights and associated constraints. This poses tractability questions around choosing the 'right' protection levels for the various flights. Because every possible combination of protection levels across flights cannot be tried for tractability reasons, we run our models with the same protection level parameters for all flights.

We now discuss the solution performance. While we discuss this with respect to networks $N_{1}$ and $N_{2}$, similar phenomena are observed for other networks as well. Table B.13 (in Appendix B and Figure 4 show that the $C C P_{f}$ solutions' robustness performance shows non-monotonicity. That is, for a fixed uncertainty set and with increases in $\alpha$, the solutions to $C C P_{f}$ can worsen with respect to flight on-time performance, passenger disruptions and total aircraft delay minutes. There are several reasons for this. First, we found that there exist multiple optimal solutions to this model, because of the large number of available strings. These solutions satisfy the same protection parameter values and yield the same objective function values, but use different sets of string to operate the flights. For example, optimal solutions to the $C C P_{f}$ with $\alpha_{i}=90$ for all ${ }_{775} i \in F$ can include solutions that satisfy $\alpha_{i}=90$ or $\alpha_{i}=94$ or $\alpha_{i}=96$ for all $i \in F$. All of these solutions are considered optimal by $C C P_{f}$ even though, 


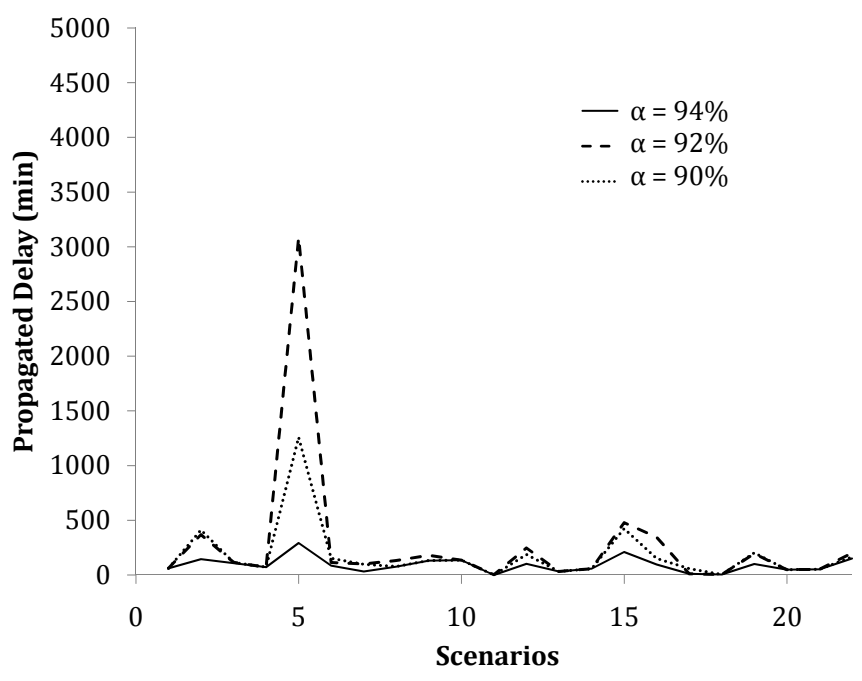

Figure 4: $C C P_{f}$ Model Solutions for Network $N_{2}(t=90)$

intuitively, the solution with the highest $\alpha$ value should be considered to be the most 'robust' among these. As a result, robustness metrics exhibit nonmonotonicity property, as shown in Table B.13. In the case of $\alpha_{i}=90$ for all $i \in F, 95.63 \%$ of the flights are delayed less than or equal to $t=90$ minutes and for $\alpha_{i}=92$ for all $i \in F, 95 \%$ of the flights are delayed less than or equal to $t$ $=90$ minutes. Similar results are observed for network $N_{1}, N_{3}$ and $N_{4}$, and for other choices of uncertainty sets represented by $t$.

Another reason for not all robustness metric values always increasing with increasing values of $\alpha$ is that the $C C P_{f}$ model focuses on selecting routings that limit the likelihood of occurrence of 'large' flight delays as a proxy for robustness, and therefore we do not expect that the solution will simultaneously optimize the three different (and sometimes conflicting) robustness metrics that we evaluate, especially those related to passenger connections.

Similarly, as illustrated in Figure 5 and in Table B.14 (in Appendix B), for the same choice of uncertainty set $t$, higher values of $\Gamma$ in $E V_{f}$ do not always produce more robust solutions. The reasons for this occurrence are similar to that for $C C P_{f}$. Multiple optimal solutions to $E V_{f}$ (and also $E V_{s}$ ) for given $\Gamma$ 


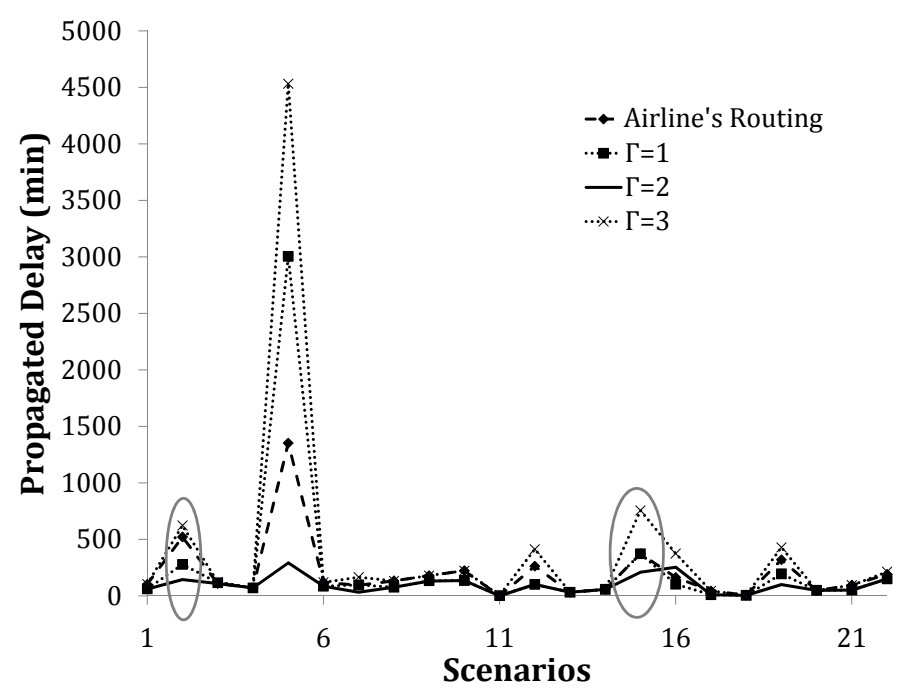

Figure 5: $E V_{f}$ Model Solutions for Network $N_{2}(t=90)$

values satisfy the protection constraints defined by $\Gamma$, even though the solutions might have very different flight strings and actual levels of slack. Hence they exhibit very different performances with respect to our robustness metrics. In fact, some of the optimal $E V_{f}$ solutions are even less robust with respect to all of our robustness metrics than the airline's routing itself, as is the case with $\Gamma=3$ in Figure 5 and Table B.14. The second reason for higher levels of $\Gamma$ in $E V_{f}$ (and $E V_{s}$ ) sometimes leading to less robust solutions is the same as that for $C C P_{f} . E V_{f}$ (and $E V_{s}$ ), like $C C P_{f}$, does not capture all the robustness metrics precisely, but rather builds robustness into the solution by ensuring that the flight legs are covered at the required service level, even in the extreme cases. Our results do, however, show slightly less variability in the alternate optima of the $E V_{s}$ model, compared to the $E V_{f}$ model, because it is based on extreme levels of propagated delay minutes for each string and accounts for every minute of delay, instead of only focusing on thresholds of $t$ minutes.

The challenge of choosing solutions with the maximum protection parameter $(\alpha$ and $\Gamma$ ) values among multiple optimal solutions needs to be addressed by more advanced models, which we present in Section 5 . 


\section{Advanced Modeling Approaches}

\subsection{Extended Chance-Constrained Programming Approach}

To overcome the limitations of the $C C P_{f}$ model discussed in Section 2.4 we propose the Extended Chance-Constrained Programming (ECCP) approach.

This method models all the protection levels $\alpha_{i}$, one for each constraint, as variables and maximizes the overall sum of protection levels across all the constraints. For a general CCP formulation such as $\max \mathbf{c}^{T} \mathbf{x}$, s.t. $P\left(\sum_{j \in J} a_{i j} x_{j} \leq\right.$ $\left.b_{i}\right) \geq \alpha_{i} \forall i \in I$, the $E C C P$ formulation would be: $\max \sum_{i \in I} \alpha_{i}$, s.t. $P\left(\sum_{j \in J} a_{i j} x_{j} \leq\right.$ $\left.b_{i}\right) \geq \alpha_{i} \forall i \in I, \mathbf{c}^{T} \mathbf{x} \geq \mathbf{c}^{T} \mathbf{z}^{*}-\delta$, where $\delta$ is the maximum allowable loss in the objective value (compared to the optimal $\operatorname{cost} \mathbf{c}^{T} \mathbf{z}^{*}$ of the nominal formulation) to attain a more robust solution. Marla (2007) and Marla (2010) discuss how this can be linearized using partial knowledge of the uncertain parameters $b_{i}$, or using suitable scenario-based approximations. We refer interested readers to Appendix C for the detailed formulation.

For aircraft routing, the protection level for each flight in constraint 16 is not specified apriori in the $E C C P$ model. Instead it is treated as a decision variable in the model. This simultaneously achieves two purposes. First, it addresses the challenge of making the combinatorial choice of protection levels, which are difficult for the user to ascertain beforehand. Instead, the protection levels in ECCP are determined internally by the model. Second, it automatically guarantees that the chosen optimal solution always satisfies the chosen protection levels exactly. We call this formulation, given by $27-(30)$, as the $\alpha-C C P_{f}$ model, which maximizes the sum of protection levels of all the constraints (flights) to determine the protection level for each flight. Therefore, relative to the benchmark $A R$ model, this model captures the probability of covering each flight by a variable $\alpha_{i}$, and maximizes the total probability of coverage of all flights. 


\section{$\alpha-\mathbf{C C P}_{\mathbf{f}}:$}

$$
\begin{aligned}
& \max \sum_{i \in F} \alpha_{i} \\
& \text { s.t. } \sum_{s \in S} a_{i s} x_{s}=1 \\
& \forall i \in F \\
& \sum_{s \in S} p_{i s} x_{s} \geq \alpha_{i} \\
& \forall i \in F
\end{aligned}
$$

Balance, Count, Non-negativity, and Integrality $(3)-7]$

Note that maximizing the sum of protection levels is just one of the multiple possible approaches for addressing the shortcomings of the basic CCP model.

Other possible approaches include: 1) maximizing a weighted sum of flight protection levels $\left(\sum_{i \in F} \alpha_{i} w_{i}\right)$ with weight $w_{i}$ assigned to flight $i$; or 2$)$ maximizing the minimum protection level $\left(\max \alpha_{\text {min }}\right)$ with constraints $\sum_{s \in S} p_{i s} x_{s} \geq \alpha_{\text {min }}$, $\forall i \in F$ replacing constraints 29 .

Yet another alternative formulation can also be obtained by minimizing the sum of expected string propagated delays for the best possible $\alpha_{i}$ values chosen by first solving the $\alpha-C C P_{f}$ model. This model is different from the other variants of $\mathrm{CCP}$ described above in that it has a string-based objective function instead of a flight-based one. We denote this formulation by $\alpha-C C P_{s}$ and is given by 31 - 34 .

$$
\begin{array}{rlr}
\alpha-\mathbf{C C P}_{\mathbf{s}}: & & \\
\min & \sum_{s \in S} d_{s} x_{s} & \\
\text { s.t. } & \sum_{s \in S} a_{i s} x_{s}=1 & \forall i \in F \\
& \sum_{s \in S} p_{i s} x_{s} \geq \alpha_{i} & \forall i \in F
\end{array}
$$

Balance, Count, Non-negativity, and Integrality $(3)-(7)$ 
Here $d_{s}=\sum_{(i, j) \in s} E\left[p d_{i j}^{s}\right]$ is defined as before. We solve this model by first solving $\alpha-C C P_{f}$ and finding the 'best' values of protection parameters, and then setting those protection parameter values in 33 to minimize the sum of expected string propagated delays (31). This formulation accounts for the fact that the $\alpha-C C P_{f}$ model itself typically has multiple optimal solutions and ensures that it picks the one with the smallest value of the sum of expected string propagated delays.

\subsection{Extended Extreme-value Robust Optimization Approach}

As with the $C C P$ model, the $E V_{f}$ model requires the specification of a parameter value (in this case, $\Gamma_{i}$ ) for each cover constraint (25). To avoid the need to repeatedly solve $E V_{f}$ to determine the best $\Gamma$ values, which is computationally extremely expensive at best and practically impossible at worst, we propose an alternative model, denoted by $\Delta-E V$, in which the $\Gamma$ parameters are modeled as decision variables. For a general 0-1 integer programming formulation such as $\max \mathbf{c}^{T} \mathbf{x}$, s.t. $\sum_{j \in J} a_{i j} x_{j} \leq b_{i} \forall i \in I, x_{j} \in\{0,1\} \forall j \in J$, with realizations of uncertain parameters $a_{i j}$ being $\tilde{a}_{i j}$, Marla (2010) formulates the robust problem by setting the protection parameters as variables, and maximizing the sum of protection parameters, within a cost budget, expressed relative to the optimal cost $\mathbf{c}^{T} \mathbf{z}^{*}$ of the nominal problem. Relative to the benchmark $A R$ model, this model ensures that the maximum number of flights are covered when faced with extreme values of delay.

The general $\Delta-E V$ formulation of Marla 2010 is as follows: $\max \sum_{i} \Gamma_{i}$
$\max _{j}\left\{\sum_{\left.i S_{i}^{\prime} \cup\left\{t_{i}\right\}\left|S_{i}^{\prime} \subseteq J,\right| S_{i}^{\prime} \mid=\left\lfloor\Gamma_{i}\right\rfloor, t_{i} \in J \backslash S_{i}^{\prime}\right\}}\left\{\sum_{j \in S_{i}^{\prime}} \hat{a}_{i j} y_{j}+\left(\Gamma_{i}-\left\lfloor\Gamma_{i}\right\rfloor\right) \hat{a}_{i t_{i}} y_{t_{i}}\right\} \leq\right.$ $b_{i} \forall i \in I, c^{T} \mathbf{x} \geq \mathbf{c}^{T} \mathbf{z}^{*}-\delta,-y_{j} \leq x_{j} \leq y_{j} \forall j, \Gamma_{i} \geq 0 \forall i, x_{j} \in\{0,1\} \forall j \in J$. The author linearizes this by taking advantage of the 0-1 integer program setting and sorting the columns $j \in J$ associated with each constraint $i$, in increasing order of their $\hat{a}_{i j}$ values. Ties are broken arbitrarily. The formulation then selects the largest sized set of $\tilde{a}_{i j}$, in each constraint $i$, that cannot be protected against their worst-case values (that is, need to take on their nominal values for 
feasibility), and seeks to minimize the sum of the sizes of these sets across all

$\Delta-\mathbf{E V}_{\mathrm{f}}:$

$$
\begin{array}{ll}
\max & \sum_{i \in F} \sum_{s \in S} \hat{a}_{i s} x_{s} \\
\text { s.t. } & \sum_{s \in S} a_{i s} x_{s} \geq 1
\end{array} \quad \forall i \in F
$$

Balance, Count, Non-negativity, and Integrality (3) - (7)

As defined in Section 2.5, $\hat{a}_{i s}=-1$ if flight $i \in F$ in string $s \in S$ has extreme value of delay exceeding $t$ minutes, based on historical data; and $\hat{a}_{i s}=0$ otherwise. The objective (35) effectively maximizes $\sum_{i \in F} \Gamma_{i}$ or minimizes $\sum_{i \in F} \Delta_{i}$ by maximizing the total value of the protection function in 25 summed for all flights $i \in F$.

Because the extreme value robust optimization framework also allows uncertainty to be modeled in the objective function, an alternative to $E V_{f}$ and $\Delta-E V_{f}$ is to capture uncertainty in a manner similar to that of the domainspecific $L C B$ model by Lan et al. (2006), but within the extreme value frame- 
work. In comparison to the benchmark $A R$ model, this would involve protecting against the extreme values of string propagated delay while ensuring that the expected propagated delay value of the chosen strings is within a pre-specified budget. This requires a completely different definition of the uncertainty set, and its paramterization, and redefining the protection parameters, as we now describe. Capturing uncertainty via the objective can be formulated as minimizing the total amount of propagated delay when any $\Gamma$ strings in the solution experience their respective extreme propagated delay values and all other strings experience their nominal propagated delay values (that is, zero). This results in a formulation we refer to as the $E V_{s}$ model, with a complex protection function like (20). The detailed formulation is presented in the Appendix A. The difficulty with this model, again, is specifying the 'best' value of $\Gamma$. Because a value of $\Gamma$ does not explicitly indicate the overall robustness of the solution, it is difficult to specify apriori. We therefore present the $\Delta-E V_{s}$ model that finds a solution such that the sum of the extreme delay values of any subset with $\Gamma$ strings does not exceed the maximum allowable system propagated delay $D$. $D$ is a user-specified threshold on total propagated delay, obtained by analyzing the historical occurrences of propagated delays. The $\Delta-E V_{s}$ solution, therefore, is an aircraft routing that allows the largest number of strings to realize their worst-case propagated delay values without exceeding the maximum allowable system propagated delay $D$. This model, like the $\Delta-E V_{f}$, is a simplified version of the general $\Delta$ model proposed by Marla (2010).

We set to zero the nominal propagated delay value for any string $s$, and let $\hat{d}_{s}$ represent the expected extreme or worst-case value of propagated delay for string $s$, as computed using historical data. Let $\bar{S}$ be the set of strings $s \in S$ with realizations of non-zero propagated delays in the historical data, that is, with non-zero $\hat{d}_{s}$. We set $v_{s}=1$ if string $s \in S$ has to take on its nominal propagated delay value of 0 and not its worst-case (historical) value for the solution to be feasible. To maximize the size of the largest subset of strings that can realize their worst-case values and ensure feasibility, we sort the strings in increasing order of their $\hat{d}_{s}$ values such that $\hat{d}_{1} \leq \hat{d}_{2} \leq \ldots \leq \hat{d}_{|S|} \cdot \Delta$ is the 
minimum number of strings with propagated delays subject to uncertainty that must assume nominal, not worst-case, values to be feasible.

$$
\begin{aligned}
& \boldsymbol{\Delta}-\mathbf{E V}_{\mathbf{s}}: \\
& \min \Delta \\
& \text { s.t. } \sum_{s \in S} \hat{d}_{s} x_{s}-\sum_{s \in S} \hat{d}_{s} v_{s} \leq D \\
& \Delta \geq \sum_{s \in S} v_{s} \quad \forall s \in \bar{S} \\
& v_{s} \leq x_{s} \\
& \forall s \in \bar{S} \quad(41) \\
& v_{s} \leq w_{s} \\
& \forall s \in \bar{S}(42) \\
& v_{s} \geq x_{s}+w_{s}-1 \\
& \forall s \in \bar{S}(43) \\
& w_{s} \geq w_{s+1} \\
& \forall s \in|S|-|\bar{S}|+1, \ldots,|S|-1 \\
& v_{s} \in[0,1] \\
& \forall s \in S \quad(46) \\
& w_{s} \in\{0,1\} \\
& \forall s \in S
\end{aligned}
$$

Constraints $(39)$ require that the total worst-case propagated delay be limited by $D$ when any $|\bar{S}|-\Delta$ strings take on worst-case propagated delay values. $v_{s}=1$ for all strings $s \in \bar{S}$ in the solution whose delay value must be set to 0 to achieve feasibility. $w_{s}=1$ for all strings $s \in \bar{S}$ for which there exists a $k \geq s$ such that $v_{k}=1$. Inequalities (41) force $v_{s}=0$ unless string $s$ is present 940 in the solution. Inequalities 42 allow $v_{s}$ to be 1 only if $w_{s}$ is 1 . Inequalities (43) allow $v_{s}$ to be 0 only if either $w_{s}=0$ or $x_{s}=0$. Constraints 40, in combination with the objective (38) count the minimum number of strings $s \in \bar{S}$ whose coefficients must take on the nominal propagated delay value of 0 . The explanation for this constraint lies in the realization that when the strings in $S$ ${ }_{945}$ are sorted in increasing order of $\hat{d}_{s}$ values, the minimum number of coefficients that must assume nominal values to maintain feasibility is determined by forcing the strings with the smallest $\hat{d}_{s}$ values, for $s \in \bar{S}$, to have their associated $v_{s}$ 
values set to 1 if $x_{s}$ is in the solution. Constraints (44) ensure that the values of $w_{s}$ variables are non-decreasing in $s$. Constraints (46) and 477) restrict $w$ and $v$ variables to take on values of 0 or 1 only.

\section{Results}

So far in this paper we have presented three basic models and four advanced models. The basic models include the domain-specific approach $(L C B)$, the chance-constrained approach $\left(C C P_{f}\right)$ and the extreme-value approach $\left(E V_{f}\right)$; while the advanced models include the flight-based and string-based variants of the $\alpha-C C P$ model, and the flight-based and string-based variants of the $\Delta-E V$ model. Each of these seven model formulations yields an aircraft routing solution (for each parameter setting) for each of our case study networks, $N_{1}, N_{2}, N_{3}$ and $N_{4}$. In this section, we present the computational results of experiments on these seven models presented in this paper, studying similarities and differences in the obtained solutions in terms of complexity, run time and robustness as measured by our metrics. We compare these model solutions with each other and also with the routing solution actually used by the airline. We then discuss the similarities and differences between the solutions, caused by the differences between the modeling paradigms, and the manner in which they capture the underlying data distributions.

\subsection{Typical Computation Times}

Table 4 reports average computation times for network $N_{2}$ with $t=90$ and $D=350$. That is, we define a flight as being 'covered' if it is delayed by less than $t=90$ minutes representing cancelations, in all the models except $\Delta-E V_{s}$. In the $\Delta-E V_{s}$ model, we restrict total propagated delay levels in the worst case to be less than the 85th percentile propagated delay from historical data, which was 350 minutes for network $N_{2}$. (For network $N_{1}$, the 85th percentile propagated delay is 160 minutes, as seen in Tables 5 and 6) For the $C C P_{f}$ and $E V_{f}$ models, multiple iterations are required to determine the appropriate $\alpha$ and $\Gamma$ values, 


\begin{tabular}{|l|c|c|c|c|}
\hline Model & $\begin{array}{c}\text { Uncertainty set } \\
\text { Parameters }\end{array}$ & $\begin{array}{c}\text { Protection } \\
\text { Parameters }\end{array}$ & Iterations & $\begin{array}{c}\text { Run time per } \\
\text { iteration (sec) }\end{array}$ \\
\hline$A R$ & None & None & 1 & 25.14 \\
$L C B$ & None & None & 1 & 42.81 \\
$C C P_{f}$ & $t$ & $\alpha_{i} \forall i$ & 1 for each $\alpha_{i}$ & 52.30 \\
$\alpha-C C P_{f}$ & $t$ & None & 1 & 72.91 \\
$\alpha-C C P_{s}$ & $t$ & None & 1 & 60.61 \\
$E V_{f}$ & $t$ & $\Gamma_{i} \forall i$ & 1 for each $\Gamma_{i}$ & 45.33 \\
$\Delta-E V_{f}$ & $t$ & None & 1 & 42.13 \\
$\Delta-E V_{s}$ & $\mathrm{D}$ & None & 1 & 6018.39 \\
\hline
\end{tabular}

Table 4: Complexity and Run Times for Network $N_{2}$ with $t=90, D=350$

respectively. Because there is no prior indication if a particular protection level $\alpha_{i}$ or $\Gamma_{i}$ (for flight $i$ ) renders the model infeasible or improves upon the currently available routing, experimentation with different values is necessary. Therefore, for these two models, Table 4 reports the run times as the average of several runs with multiple protection parameter values. While these particular results are for network $N_{2}$ with $t=90$ and $D=350$, similar results are obtained for different choices of uncertainty sets and for network $N_{1}$ as well. Although they each had to be solved only once, the computation times of $A R, L C B, \alpha-C C P_{f}$, $\alpha-C C P_{s}$, and $\Delta-E V_{f}$ models are found to be comparable to the time required to solve a single iteration of $C C P_{f}$ and $E V_{f}$. On the other hand, $\Delta-E V_{s}$ has a much larger run time due to the large number of routes/strings, because the model has the number of constraints equal to the number of strings. Therefore, we should be cautious while using it in an even larger network, as the number of constraints grows exponentially with the number of flights.

\subsection{Solution Differences due to Modeling Paradigms}

In this section, we compare the quality of solutions from all the models in terms of three evaluation metrics: on-time performance, total propagated delay 


\begin{tabular}{|c|cccccc|}
\hline & \multicolumn{5}{|c|}{ Flight On-time Performance } \\
\hline & $\leq 15 \mathrm{~min}$ & $\leq 30 \mathrm{~min}$ & $\leq 60 \mathrm{~min}$ & $\leq 90 \mathrm{~min}$ & $\leq 120 \mathrm{~min}$ & $\leq 180 \mathrm{~min}$ \\
\hline Airline's Routing & $82.61 \%$ & $91.21 \%$ & $96.37 \%$ & $98.00 \%$ & $98.80 \%$ & $99.44 \%$ \\
$L C B$ & $89.63 \%$ & $95.39 \%$ & $98.36 \%$ & $99.18 \%$ & $99.55 \%$ & $99.82 \%$ \\
$C C P_{f}$ & $84.36 \%$ & $92.71 \%$ & $97.24 \%$ & $98.56 \%$ & $99.16 \%$ & $99.63 \%$ \\
$\alpha-C C P_{f}$ & $90.89 \%$ & $95.87 \%$ & $98.50 \%$ & $99.24 \%$ & $99.59 \%$ & $99.83 \%$ \\
$\alpha-C C P_{s}$ & $89.60 \%$ & $95.37 \%$ & $98.35 \%$ & $99.18 \%$ & $99.55 \%$ & $99.82 \%$ \\
$E V_{f}$ & $83.68 \%$ & $93.04 \%$ & $97.37 \%$ & $98.61 \%$ & $99.19 \%$ & $99.63 \%$ \\
$\Delta-E V_{f}$ & $90.89 \%$ & $95.87 \%$ & $98.50 \%$ & $99.24 \%$ & $99.59 \%$ & $99.83 \%$ \\
$\Delta-E V_{s}$ & $82.76 \%$ & $91.42 \%$ & $96.47 \%$ & $98.06 \%$ & $98.84 \%$ & $99.46 \%$ \\
\hline
\end{tabular}

Table 5: On-time Performance Results for Network $N_{1}$ with $t=90, D=160$

and passenger disruptions. The definitions of these metrics and evaluation setup are as described in Section 3.4 . We first discuss the results for networks $N_{1}$ and $N_{2}$, for the sake of clarity. Because the results for $N_{3}$ and $N_{4}$ are similar, we present them after discussing $N_{1}$ and $N_{2}$.

At this juncture, it is worth reviewing our first result. As noted in Section 4, the original $C C P_{f}$ and $E V_{f}$ models exhibit performance that does not improve monotonically with increasing values of the associated protection parameters. This is because these models do not choose solutions that are on the pareto-frontier with respect to the protection parameters. While a similar result has been discussed by Iancu and Trichakis (2014), this is the first datadriven evidence for large-scale network-based problems, in which the complexity is compounded by the user needing to choose among a combinatorially growing set of protection parameters.

Our second major result is that both the $\Delta-E V_{f}$ and $\alpha-C C P_{f}$ model solutions improve upon the airline's routing, unlike the solutions generated by the $E V_{f}$ model which did not outperform the airline's routing with respect to some metrics. In general, the extended models (with the exception of $\Delta-E V_{s}$ ) perform better than the original ones. Table 5 and Table 6 list the on-time performance, total propagated delay and passenger disruption metrics for net- 


\begin{tabular}{|c|c|c|}
\hline & Propagated delay & Pax Disruptions \\
\hline & Total average (min) & \% Disrupted pax \\
\hline Airline's Routing & 95.90 & $6.50 \%$ \\
$L C B$ & 61.88 & $4.40 \%$ \\
$C C P_{f}$ & 81.35 & $5.74 \%$ \\
$\alpha-C C P_{f}$ & 62.35 & $3.94 \%$ \\
$\alpha-C C P_{s}$ & 61.87 & $4.40 \%$ \\
$E V_{f}$ & 107.06 & $4.68 \%$ \\
$\Delta-E V_{f}$ & 61.43 & $3.94 \%$ \\
$\Delta-E V_{s}$ & 93.85 & $6.46 \%$ \\
\hline
\end{tabular}

Table 6: Propagated Delay and Passenger Disruption Improvements in $N_{1}$ with $t=90, D=$ 160

work $N_{1}$. Note that for $C C P_{f}$ and $E V_{f}$, the tables report the 'best' solutions obtained over multiple parameter values, chosen using the five-fold cross validation approach. For $N_{1}$, we notice that $\alpha-C C P_{f}$, and $\Delta-E V_{f}$ perform the

best overall across the various robustness metrics. $\alpha-C C P_{s}$ and $L C B$ perform second best in terms of our metrics of interest. $C C P_{f}, E V_{f}$ and $\Delta-E V_{s}$ perform poorly compared to the other models, but still are somewhat better than the routing solution that was actually used by the airline. Table 7 and Table 8 present the same set of performance metrics for network $N_{2}$. For $N_{2}$, we notice that $\alpha-C C P_{s}$ performs the best overall across these different metrics. Also, $L C B, C C P_{f}, \alpha-C C P_{f}$ and $\Delta-E V_{f}$ continue to perform well. On the other hand, as in case of network $N_{1}$, the $E V_{f}$ and $\Delta-E V_{s}$ models perform poorly compared to other models, but nevertheless improve slightly upon the routing actually used by the airline. Thus, our third major result is that while $\Delta-E V_{f}$, $\alpha-C C P_{f}$, and $\alpha-C C P_{s}$ are the best performers across the different metrics and across different networks, the performance of the basic models $C C P_{f}$ and $L C B$ (with $C C P_{f}$ 's best parameter settings) is only marginally worse. However, $C C P_{f}$ model along with $E V_{f}$ and $\Delta-E V_{s}$ models encounters issues of tractabil- 
ity, and $C C P_{f}$ and $E V_{f}$ models also suffer from the difficulty in specifying the 'right' values of the protection parameters. Instead, the extended models allow us to find the 'best' levels of robustness parameters. The solutions obtained by these extended models are robust and consistently improve upon the airline's routing with respect to on-time performance metrics, total propagated delays and passenger disruption metrics.

We now compare the performance of the flight-based models $\alpha-C C P_{f}$ and $\Delta-E V_{f}$ with the string-based models $\alpha-C C P_{s}$ and $\Delta-E V_{s}$, to arrive at our fourth major result. From this point onward, we compare also the results from networks $N_{3}$ and $N_{4}$ as we are discussing the performance of the advanced modeling approaches we propose in this paper. From Tables 5 - 12 we see that the models that capture delays at the flight level, by capturing uncertainty in the parameters $a_{i s}$, perform somewhat better than those that capture uncertainty at an aggregate string-level through the $d_{s}$ parameters. The only exception is that for network $N_{2}$, where $\alpha-C C P_{f}$ slightly outperforms $\alpha-C C P_{s}$. This is because these flight-level models $\alpha-C C P_{f}$ and $\Delta-E V_{f}$ control delay at the individual flight level, compared to the $\alpha-C C P_{s}$ and $\Delta-E V_{s}$ models. Between two strings with similar total propagated delay but different levels of flight delays, the flight-based models choose the string with lower individual flight delays. Therefore they improve flight on-time performance, allow better connections for passengers, and hence perform better than the string-based models.

Finally, we compare the performances of the flight-based and string-based models for the four networks $N_{1}, N_{2}, N_{3}$ and $N_{4}$, to arrive at our fifth major result. As mentioned earlier, the overall performance of the results from the flight-based models is better than those of the string-based models. This phenomenon is very clear when the performance is evaluated on network $N_{1}$, which is based on distribution-fitting-approach and thus assumes that the independent delays of different flights are statistically uncorrelated. However, our results show that the relative performance of both the string-based models $\left(\alpha-C C P_{s}\right.$ and $\left.\Delta-E V_{s}\right)$ compared with those of the flight-based counterparts $\left(\alpha-C C P_{f}\right.$ and $\left.\Delta-E V_{f}\right)$ improves for networks $N_{2}$ and $N_{3}$. In fact, $\alpha-C C P_{s}$ 


\begin{tabular}{|c|cccccc|}
\hline & \multicolumn{5}{|c|}{ Flight On-time Performance } \\
\hline & $\leq 15 \mathrm{~min}$ & $\leq 30 \mathrm{~min}$ & $\leq 60 \mathrm{~min}$ & $\leq 90 \mathrm{~min}$ & $\leq 120 \mathrm{~min}$ & $\leq 180 \mathrm{~min}$ \\
\hline Airline's Routing & $72.92 \%$ & $81.40 \%$ & $89.45 \%$ & $93.66 \%$ & $96.23 \%$ & $98.58 \%$ \\
$L C B$ & $73.43 \%$ & $81.92 \%$ & $89.90 \%$ & $93.95 \%$ & $96.46 \%$ & $98.63 \%$ \\
$C C P_{f}$ & $73.61 \%$ & $82.01 \%$ & $89.90 \%$ & $93.91 \%$ & $96.37 \%$ & $98.60 \%$ \\
$\alpha-C C P_{f}$ & $73.79 \%$ & $82.07 \%$ & $89.96 \%$ & $93.96 \%$ & $96.44 \%$ & $98.62 \%$ \\
$\alpha-C C P_{s}$ & $74.55 \%$ & $82.82 \%$ & $90.41 \%$ & $94.27 \%$ & $96.63 \%$ & $98.68 \%$ \\
$E V_{f}$ & $72.73 \%$ & $81.33 \%$ & $89.48 \%$ & $93.66 \%$ & $96.25 \%$ & $98.58 \%$ \\
$\Delta-E V_{f}$ & $73.54 \%$ & $81.80 \%$ & $89.75 \%$ & $93.83 \%$ & $96.31 \%$ & $98.55 \%$ \\
$\Delta-E V_{s}$ & $73.55 \%$ & $81.98 \%$ & $89.90 \%$ & $93.92 \%$ & $96.43 \%$ & $98.62 \%$ \\
\hline
\end{tabular}

Table 7: On-time Performance Results for Network $N_{2}$ with $t=90, D=350$

turns out to be the overall best model for networks $N_{2}$ and $N_{3}$. This can be explained by the fact that there are significant correlations between the independent flight delays in these networks and capturing uncertainty at the string levels allows the string-models to account for such correlations between the independent flight delays. Flight-based models do not have this ability and hence perform slightly worse for network $N_{2}$ and $N_{3}$ than for network $N_{1}$.

\subsection{Influences of Underlying Data Distributions}

We now discuss the influence of the underlying data distributions on the efficacy of each of the modeling paradigms. Because the $\Delta-E V_{f}$ and $\Delta-E V_{s}$ models are distribution-free while the $\alpha-C C P_{f}$ and $\alpha-C C P_{s}$ models are distribution-based, the differences in their efficacy are also expected to depend on the underlying data distributions.

To illustrate this point, consider Figure 6 in which coverage probabilities of two flight-string combinations $i_{1} s_{1}$ and $i_{2} s_{2}$ are shown. $\Delta-E V_{f}$ and $E V_{f}$ do not distinguish between both these realizations, because both $i_{1} s_{1}$ and $i_{2} s_{2}$ have non-zero historical probability of realizing a delay value greater than $t$. On the other hand, the $\alpha-C C P_{f}, \alpha-C C P_{s}$ and $C C P_{f}$ distinguish between these realizations in a probabilistic manner.

Related to the string-based models $\Delta-E V_{s}$ and $\alpha-C C P_{s}$, we find that the 


\begin{tabular}{|c|c|c|}
\hline & Propagated delay & Pax Disruptions \\
\hline & Total average (min) & \% Disrupted pax \\
\hline Airline's Routing & 458.59 & $6.35 \%$ \\
$L C B$ & 412.77 & $5.53 \%$ \\
$C C P_{f}$ & 342.26 & $4.89 \%$ \\
$\alpha-C C P_{f}$ & 394.61 & $5.01 \%$ \\
$\alpha-C C P_{s}$ & 342.56 & $5.19 \%$ \\
$E V_{f}$ & 443.18 & $6.45 \%$ \\
$\Delta-E V_{f}$ & 371.25 & $5.04 \%$ \\
$\Delta-E V_{s}$ & 516.15 & $6.39 \%$ \\
\hline
\end{tabular}

Table 8: Propagated Delay and Passenger Disruption Improvements in $N_{2}$ with $t=90, D=$ 350

\begin{tabular}{|c|cccccc|}
\hline & \multicolumn{5}{|c|}{ Flight On-time Performance } \\
\hline & $\leq 15 \mathrm{~min}$ & $\leq 30 \mathrm{~min}$ & $\leq 60 \mathrm{~min}$ & $\leq 90 \mathrm{~min}$ & $\leq 120 \mathrm{~min}$ & $\leq 180 \mathrm{~min}$ \\
\hline Airline's Routing & $75.61 \%$ & $82.90 \%$ & $85.00 \%$ & $91.72 \%$ & $92.23 \%$ & $94.33 \%$ \\
$L C B$ & $80.76 \%$ & $86.20 \%$ & $89.50 \%$ & $94.00 \%$ & $95.60 \%$ & $97.44 \%$ \\
$\alpha-C C P_{f}$ & $84.20 \%$ & $86.90 \%$ & $88.45 \%$ & $94.89 \%$ & $98.48 \%$ & $98.00 \%$ \\
$\alpha-C C P_{s}$ & $84.50 \%$ & $87.24 \%$ & $89.56 \%$ & $95.23 \%$ & $98.20 \%$ & $98.50 \%$ \\
$\Delta-E V_{f}$ & $84.60 \%$ & $86.92 \%$ & $88.38 \%$ & $94.70 \%$ & $98.70 \%$ & $98.90 \%$ \\
$\Delta-E V_{s}$ & $83.95 \%$ & $84.70 \%$ & $87.30 \%$ & $93.40 \%$ & $96.83 \%$ & $98.20 \%$ \\
\hline
\end{tabular}

Table 9: On-time Performance Results for Network $N_{3}$ with $t=60, D=176$ 


\begin{tabular}{|c|c|c|}
\hline & Propagated delay & Pax Disruptions \\
\hline & Total average (min) & \% Disrupted pax \\
\hline Airlines Routing & 195.29 & $5.20 \%$ \\
$L C B$ & 186.49 & $3.62 \%$ \\
$\alpha-C C P_{f}$ & 175.94 & $3.56 \%$ \\
$\alpha-C C P_{s}$ & 170.02 & $3.64 \%$ \\
$\Delta-E V_{f}$ & 173.89 & $3.57 \%$ \\
$\Delta-E V_{s}$ & 183.62 & $3.74 \%$ \\
\hline
\end{tabular}

Table 10: Propagated Delay and Passenger Disruption Improvements in $N_{3}$ with $t=60, D$ $=176$

\begin{tabular}{|c|cccccc|}
\hline & \multicolumn{5}{|c|}{ Flight On-time Performance } \\
\hline & $\leq 15 \mathrm{~min}$ & $\leq 30 \mathrm{~min}$ & $\leq 60 \mathrm{~min}$ & $\leq 90 \mathrm{~min}$ & $\leq 120 \mathrm{~min}$ & $\leq 180 \mathrm{~min}$ \\
\hline Airline's Routing & $78.76 \%$ & $83.48 \%$ & $86.00 \%$ & $90.90 \%$ & $95.40 \%$ & $97.85 \%$ \\
$L C B$ & $82.28 \%$ & $86.57 \%$ & $88.00 \%$ & $89.70 \%$ & $90.76 \%$ & $95.20 \%$ \\
$\alpha-C C P_{f}$ & $86.00 \%$ & $89.50 \%$ & $92.20 \%$ & $94.50 \%$ & $95.49 \%$ & $98.35 \%$ \\
$\alpha-C C P_{s}$ & $85.71 \%$ & $88.65 \%$ & $91.43 \%$ & $93.64 \%$ & $94.31 \%$ & $98.10 \%$ \\
$\Delta-E V_{f}$ & $87.00 \%$ & $90.76 \%$ & $93.75 \%$ & $95.29 \%$ & $96.20 \%$ & $98.79 \%$ \\
$\Delta-E V_{s}$ & $84.80 \%$ & $87.90 \%$ & $90.92 \%$ & $93.45 \%$ & $95.37 \%$ & $98.35 \%$ \\
\hline
\end{tabular}

Table 11: On-time Performance Results for Network $N_{4}$ with $t=60, D=210$

\begin{tabular}{|c|c|c|}
\hline & Propagated delay & Pax Disruptions \\
\hline & Total average (min) & \% Disrupted pax \\
\hline Airline's Routing & 155.01 & $5.53 \%$ \\
$L C B$ & 136.51 & $4.92 \%$ \\
$\alpha-C C P_{f}$ & 131.30 & $4.56 \%$ \\
$\alpha-C C P_{s}$ & 126.89 & $4.60 \%$ \\
$\Delta-E V_{f}$ & 127.76 & $4.60 \%$ \\
$\Delta-E V_{s}$ & 137.02 & $4.78 \%$ \\
\hline
\end{tabular}

Table 12: Propagated Delay and Passenger Disruption Improvements in $N_{4}$ with $t=60, D$ $=210$ 


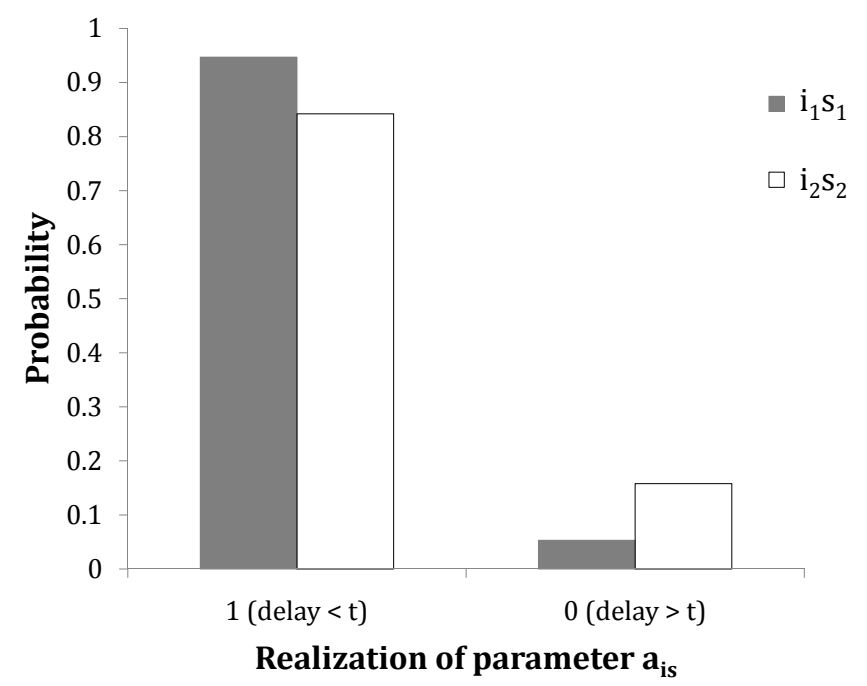

Figure 6: The Realization Probabilities of $a_{i s}$ in Network $N_{2}$

string delays often follow a bimodal distribution. As an illustration, consider Figure 7(a) in which the distributions of propagated delays for strings in network $N_{1}$ are shown. The propagated delay distribution is seen to be roughly bi-modal, with a large ( $88 \%$ to $95 \%$ ) probability of the propagated string delay being on the lower end of the scale, and a small ( $5 \%$ to $12 \%$ ) probability of the propagated delay being close to its worst-case value. The probability of occurrence of interim values is relatively small. This causes the average value and worst-case value of string propagated delays to be as shown in Figure 7(b)

The $L C B$ model uses average values of string propagated delay, shown in Fig. 7(b). Because the average is typically at around the 85th or 90th percentile of string propagated delay, $L C B$ effectively ignores the extreme value occurrences forming the remaining $5-10 \%$ of the distribution. Its objective of minimizing the expected value of the total propagated delay seems to correlate well with our robustness metrics on average, with the $L C B$ solutions performing well on all metrics. Although the $L C B$ model does not capture the probability distribution of delays, by using average delay values, it effectively behaves like a special case of the string-based $\alpha-C C P_{s}$ model where the quantiles of $d_{s}$ in the objective 


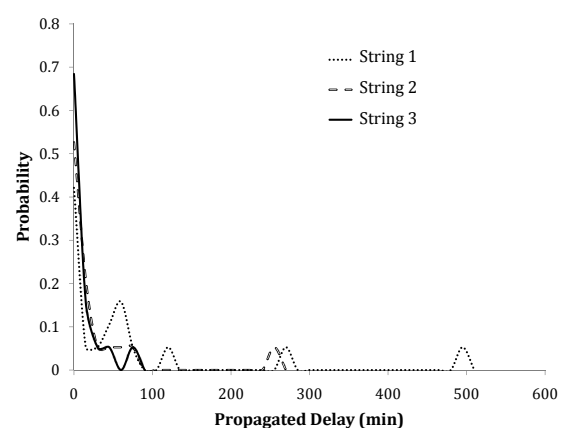

(a) String Propagated Delays

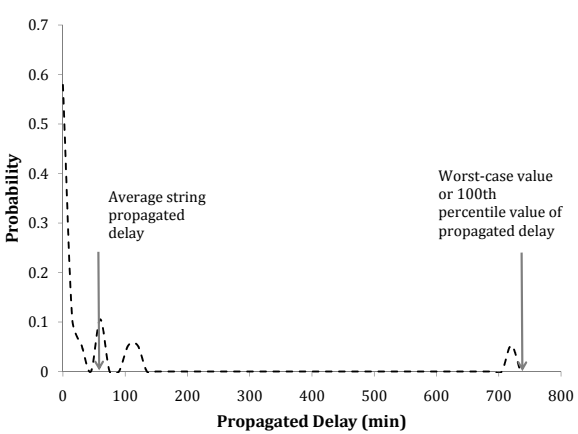

(b) Average and Extreme Values of String Propagated Delays

Figure 7: Propagated Delay Distributions of Strings

function of $\alpha-C C P_{s}$ model are set, for these problems, to approximately the 90th percentile level. The advantage of $L C B$ over $\alpha-C C P_{s}$ is that it is able to capture uncertainty in a simpler way, resulting in a highly tractable model; while its disadvantage is that the resulting solutions are overall slightly less models is very similar to those of $L C B$ and $\alpha-C C P_{f}$ models; but additionally there also exist multiple alternate optima for $\Delta-E V_{f}$ that perform very differently. Depending on the solution output provided by the optimization solver being used, these models can have significantly varying performances. Simi- 
larly, the $\Delta-E V_{s}$ model is based on the extreme values of string propagated delays. This emphasis on extreme values of delays does not necessarily drive the extreme-value models towards good values of the average-case robustness metrics. Because the $\Delta-E V_{f}$ and $\Delta-E V_{s}$ models are formulated to avoid low probability situations involving worst-case delays or non-coverage, optimal solutions can have widely varying values of average performance as measured by average on-time performance, average propagated delays, and average number of disrupted passengers.

We can apply the insights gained in solving the aircraft routing problem with $L C B, \alpha-C C P_{f}$ and $\alpha-C C P_{s}$ models to improve the extreme valuebased robust optimization models. Specifically, by adjusting the uncertainty set definition or 'extreme' values in the $\Delta-E V_{s}$ model to, say, capture additional quantiles of string propagated delay, we can generate solutions using these models that perform closer to those of the $L C B, \alpha-C C P_{f}$ and $\alpha-C C P_{s}$ models. This, of course, underscores the difficulty of setting these uncertainty sets a priori, and the sensitivity of the solutions to these model inputs.

\section{Conclusions}

In this paper, we study the application of three types of models - domainspecific, probability distribution-free and probability-distribution based - to the problem of aircraft routing. These three approaches lead to different models with different solutions which have different robustness performances with respect to various metrics of interest.

The probability distribution-free extreme-value models $\left(E V_{f}\right.$ and $\left.E V_{s}\right)$ were based on the Bertsimas and Sim robust optimization approach Bertsimas and Sim, 2004, 2003). The probability distribution-based models are based on

${ }_{1140}$ Charnes and Cooper's chance-constrained programming approach Charnes and Cooper, 1959, 1963). Finally, the domain-specific model ( $L C B)$ was Lan, Clarke and Barnhart's (Lan et al., 2006) robust aircraft routing approach. Significantly increased complexity and solution times are associated with the extreme-value 
$(E V)$ and chance-constrained programming $(C C P)$ models, when compared

performance, on average, than string-level (route-level) robust models such as $\alpha-C C P_{s}$ and $\Delta-E V_{s}$. This is because the former two models allow for a larger degree of control at the flight-level as opposed to the string-based models that constrain the propagated delays only at the string-level. This ensures both 
tractable formulations as well as superior solutions. String-level models (especially the $\alpha-C C P_{s}$ model) do a better job of capturing the correlations between independent flight delays and thus have an improved (robustness) performance when independent delays are correlated. Fourth, the extreme-value based models including the $\Delta-E V_{f}$ and $\Delta-E V_{s}$ models, in most cases, exhibit significant variability in performance due to their focus on the worst-case values of delays. Like the $E V$ model, they can often have multiple optimal solutions. Although alternative optimal solutions have the same objective function value, they differ significantly in terms of the associated values of the various robustness metrics. Our findings show that extreme-value based models $E V_{f}, \Delta-E V_{f}$ and $\Delta-E V_{s}$ generate solutions with variable performance levels; varying from good improvement compared to the airline's routing, to no significant improvement or even deterioration. This behavior is because the mechanism used for capturing robustness $(\Gamma$ or $\Delta)$ is driven by extreme values of delay. This dependence on extreme delay values, ignoring probabilistic information, leads in some cases to a large disparity in the performance of the alternative optimal solutions. In such cases, extra care should be taken in evaluating alternative optimal solutions to these approaches. Thus, we conclude that it is not effective to drive the solution process with extreme values when these extreme values are rare.

The domain-specific approach and the probability distribution-based approaches are very similar in that expected values of string propagated delay, used to drive the $L C B$ model, are typically found to be at around the 85 th or 90th percentile of the string propagated delay distribution; and hence cause the formulation to focus on higher-probability events. Similarly, probability distribution-based approaches also focus on higher-probability delay events, and produce improved routings according to our metrics. These approaches thus capture more information about the system and focus on more-likely delay events, and thus are more in line with our metrics of interest. Though the domain-specific approach in itself does not explicitly capture knowledge of probability distributions, by implicitly incorporating some of the relevant delay quantiles in its objective it can achieve improved results through a less complex 
model. The probability distribution-based approaches allow more fine-tuning of robustness using the $\alpha$ and $t$ parameters, but come at a cost of creating a larger, more complex formulation (albeit very tractable for this application) and the added implementation cost, compared to $L C B$, due to the requirement of knowing the delay distributions.

In conclusion, our paper highlights that the efficacy of any given robust approach is determined not by the approach or model alone, but by the interaction between the model, data (including network structure and delay patterns), and evaluation metrics. Thus, an opportunity for future extension of this analysis is to test our comparison framework through controlled experiments on several networks with varying characteristics, such as hub-and-spoke or point-to-point network structure, and specific delay patterns and (or) correlations, in order to see how each model would perform under each setting.

Our paper therefore, underscores the importance of choosing an approach that aligns itself well with the data distributions for the aircraft routing problem and metrics of interest to the DoT, industry and passengers. When applying general robust approaches to more specific problems, care should be taken to understand the nature of uncertainty and to choose robustness parameters in relation to the metrics, especially when the metrics affect multiple stakeholders. 


\section{Appendix A. $E V_{s}$ Model Formulation}

The $E V_{s}$ model plans for the case when a user-defined number of strings $\Gamma$ in the formulation realize their worst-case values of propagated delay, while other strings do not vary from their assumed deterministic or nominal delay values (equal to zero).

The protection parameter $\Gamma$ is defined in the interval $[0,|S|]$. Let $\hat{d}_{s}$ be the worst-case (maximum or 100th percentile) value of propagated delay of string $s$ observed in the historical data. Note that this is not the worst possible realization of propagated delay, but it is the worst-case actual realization in the selected period of historical data.

The formulation minimizes the extreme (or worst-case) value of string propagated delay caused by any set of $\Gamma$ strings in the solution realizing their worstcase values, and the other strings attaining their nominal values of propagated delay (equal to zero). That is, it minimizes the maximum propagated delay caused by any subset of $\Gamma$ strings in the solution realizing their extreme propagated delay values, as shown in A.1. Variable $w_{s}$ for string $s \in S$ takes on value 1 if string $s$ is present in the solution, and the model protects against its extreme value being realized. The extreme-value formulation, according to Bertsimas and Sim (Bertsimas and Sim, 2003), is as follows.

$$
\min \left\{\sum_{s \in S} 0 x_{s}+\max _{\left\{S^{\prime} \cup\{t\}\left|S^{\prime} \subseteq S,\right| S^{\prime} \mid=\lfloor\Gamma\rfloor, t \in S \backslash S^{\prime}\right\}}\left\{\sum_{s \in S^{\prime}} \hat{d}_{s} w_{s}+(\Gamma-\lfloor\Gamma\rfloor) \hat{d}_{t} w_{t}\right\}\right\}
$$

s.t. Cover, Balance, Count, Non-negativity, and Integrality (2) - 7)

$$
\begin{aligned}
& x_{s} \leq w_{s} \quad \forall s \in S \\
& w_{s} \geq 0 \quad \forall s \in S
\end{aligned}
$$

ㅁ This formulation can be linearized using the process described in Bertsimas

1245 and Sim (2004) and Marla (2010), and cast as a mixed integer program, as follows: 
$\mathbf{E V}_{\mathrm{s}}$ :

$$
\begin{aligned}
& \min z \Gamma+\sum_{s \in S} p_{s} \\
& \text { s.t. } z+p_{s} \geq \hat{d}_{s} w_{s} \quad \forall s \in S
\end{aligned}
$$

Cover, Balance, Count, Non-negativity, and Integrality (2) - (7) (A.7)

$$
\begin{array}{ll}
x_{s} \leq w_{s} & \forall s \in S \\
w_{s} \geq 0 & \forall s \in S \\
z \geq 0 . &
\end{array}
$$

These mixed integer programming formulations lose the special set partitioning structure of the original $A R$ formulation (Marla, 2007) due to the type of constraints added to the formulation, and face computational challenges (see Section 6.1).

\section{Appendix B. Tables for Preliminary Results - Section 4}

\begin{tabular}{l|ccccc|cc}
\hline & \multicolumn{3}{|c|}{ \% Flight Delay (min) improvements } & \multicolumn{2}{c}{ Pax Disruptions } \\
\hline & $\leq 15$ & $\leq 60$ & $\leq 90$ & $\leq 120$ & $\leq 180$ & \#D-pax & D-pax reduced \\
\hline$\alpha_{i}=90 \forall i$ & 78.54 & 93.10 & 95.63 & 97.82 & 98.91 & 1025 & 6.8 \\
$\alpha_{i}=92 \forall i$ & 77.54 & 92.54 & 95.00 & 97.36 & 98.54 & 1209 & -9.9 \\
$\alpha_{i}=94 \forall i$ & 79.54 & 93.73 & 96.00 & 98.18 & 99.18 & 987 & 10.2 \\
Airline's Routing & 77.72 & 92.82 & 95.30 & 97.73 & 98.91 & 1100 & 0.0 \\
\hline
\end{tabular}

Table B.13: Non-Monotonicity of Robustness Metrics for Network $N_{2}$ for the $C C P_{f}$ Model $(t$ $=90)$ 


\begin{tabular}{c|cccc|cc}
\hline & \multicolumn{4}{|c|}{ Flight Delays } & \multicolumn{2}{c}{ Pax Disruptions } \\
\hline & $\leq 15$ min & $\leq 60$ min & $\leq 120$ min & $\leq 180$ min & \#D-pax & D-pax reduced \\
\hline$\Gamma_{i}=1 \forall i$ & 78.63 & 93.00 & 97.36 & 98.54 & 1222 & -11.0 \\
$\Gamma_{i}=2 \forall i$ & 79.54 & 93.54 & 98.09 & 99.18 & 987 & 10.3 \\
$\Gamma_{i}=3 \forall i$ & 74.18 & 91.00 & 96.54 & 97.90 & 1353 & -23.0 \\
Airline's Routing & 77.72 & 92.82 & 97.73 & 98.91 & 1100 & 0.0 \\
\hline
\end{tabular}

Table B.14: Non-Monotonicity of Robustness Metrics for Network $N_{2}$ for the $E V_{f}$ Model $(t$ $=90)$

\section{Appendix C. Extended Chance-Constrained Programming - Gen- eral Formulation}

The standard linear optimization problem that is required to be made robust 1255 is:

$$
\begin{array}{cc}
\max & \mathbf{c}^{T} \mathbf{x} \\
\text { s.t. } & \sum_{j \in J} a_{i j} x_{j} \leq b_{i} \quad \forall i \in I \\
\mathbf{x} \geq 0,
\end{array}
$$

where $I$ is the set of constraints, and $J$ the set of variables, $c_{j}$ is the profit coefficient for variable $j$ and $b_{i}$ is the right-hand side value for constraint $i$, $\forall i \in I . a_{i j}$ for all $i \in I, j \in J$ is the coefficient of variable $j$ in constraint $i$. Assume that the uncertainty is in the right hand side parameters $b_{i}$ and in the objective function coefficients $c_{j}$. Charnes and Cooper (1963) write the general chance-constrained formulation as follows:

$$
\begin{array}{cc}
\max & f(\mathbf{c}, \mathbf{x}) \\
\text { s.t. } & P\left(\sum_{j \in J} a_{i j} x_{j} \leq b_{i}\right) \geq \alpha_{i} \quad \forall i \in I \\
\mathbf{x} \geq 0,
\end{array}
$$

where ' $\mathrm{P}$ ' indicates 'probability', and $f$ could be expected value-based, variancebased or probabilistic forms in the objective. The most common form for $f$ is 
the expected value. $\alpha_{i}\left(0 \leq \alpha_{i} \leq 1\right)$ for all $i$, is a user-specified protection level for constraint $i$, and $\left(1-\alpha_{i}\right)$ specifies the maximum probability of violation of constraint $i$.

Ben-Israel (Ben-Israel, 1962) shows that the chance-constraint (C.5) can be linearized as follows:

$$
P\left(\sum_{j \in J} a_{i j} x_{j} \leq b_{i}\right) \geq \alpha_{i} \Leftrightarrow \sum_{j \in J} a_{i j} x_{j} \leq F_{b_{i}}^{-1}\left(1-\alpha_{i}\right),
$$

with $y=F_{b_{i}}^{-1}\left(1-\alpha_{i}\right)$ equal to the quantile value in the cumulative distribution function $(\mathrm{CDF}), F_{b_{i}}$, of $b_{i}$ such that the probability that $b_{i}$ takes on values less than or equal to $y$ is $\left(1-\alpha_{i}\right)$. That is, if $f\left(b_{i}\right)$ is the probability density function of $b_{i}, \int_{-\infty}^{y} f\left(b_{i}\right) d b_{i}=1-\alpha_{i}$.

In Marla (2010), to avoid the need to specify the protection level for each constraint explicitly, the author includes a constraint on the overall expected profit of the robust solution (similar to D.4 and changes the objective to one of maximizing the sum of protection levels provided for all constraints, as follows:

where $\mathbf{c}^{T} \mathbf{z}^{*}$ is the profit of the nominal optimal solution $\mathbf{z}^{*}$ to C.1 -C.3, found using nominal values of the $\mathbf{b}$ and $\mathbf{c}$ parameters.

To linearize (??) - (??), we require the knowledge of some quantiles of the right-hand-side for each constraint, and the expected values of the profit coefficients. Let $b_{i}$ be the nominal value for the right-hand-side of the $i$ th constraint, and $c_{j}$ be the expected profit coefficient corresponding to the $j$ th variable, for all $j \in J$. Let $K_{i}$ represent the set of discretized protection levels known for $b_{i}$. We now set the protection levels $\alpha_{i}$ as variables, representing the quantile that ${ }_{1285}$ is chosen from among the $\left\|K_{i}\right\|$ available quantiles. Let $b_{i}^{k}$ be the $k$ th quantile value of the right-hand-side (RHS) parameter of the $i$ th constraint, for all $k \in K_{i}, i \in I$; and $z_{j}^{*} \forall j \in J$ be the nominal optimal solution to C.1 - C.3. $p_{i}^{k}$ is the protection probability associated with quantile $k \in K_{i}$ for constraint 
$i \in I$. The objective function equals the sum of protection levels achieved over all constraints $i \in I$. To capture the tradeoff of robustness with profits, we assume that the planner is willing to forego a (user-specified) profit $\Delta$ to instead achieve a robust plan.

Decision variables $y_{i}^{k}$ are binary variables that equal 1 if the protection level (expressed as a probability $p_{i}^{k}$, with $0 \leq p_{i}^{k} \leq 1$ ) represented by the $k$ th quantile ( $k \in K_{i}$ ) is attained in constraint $i \in I$; and 0 otherwise. Note that if the $k$ th quantile value is protected against, the $(k+1)$ st quantile is also automatically protected against. This is ensured by the fact that constraints C.11 are "less than or equal to" inequalities. The $y_{i}^{k}$ values for any constraint $i$, follow a step function. Variables $\alpha_{i}$ represent the protection level attained for the $i$ th constraint, for all $i \in I$.

The extended chance-constrained model (ECCP) is as follows:

$$
\begin{array}{lr}
\max \sum_{i \in I} \alpha_{i} & \\
\text { s.t. } \sum_{j \in J} c_{j} x_{j} \geq \sum_{j \in J} c_{j} z_{j}^{*}-\Delta & \\
& \sum_{j \in J} a_{i j} x_{j} \leq \sum_{k=1}^{K} b_{i}^{k}\left(y_{i}^{k}-y_{i}^{k-1}\right) \\
& \\
y_{i}^{k} \geq y_{i}^{k-1} & \forall i \in I \\
y_{i}^{0}=0 & \forall k=1, \ldots, K_{i}, i \in I, \\
y_{i}^{K}=1 & \forall i \in I \\
\alpha_{i} \leq \sum_{k=1}^{K_{i}} p_{i}^{k}\left(y_{i}^{k}-y_{i}^{k-1}\right) & \forall i \in I \\
x_{j} \geq 0 & \forall i \in I \\
y_{i}^{k} \in\{0,1\} & \forall j \in J \\
0 \leq \alpha_{i} \leq 1 & \forall k \in K_{i}, i \in I \\
& \forall i \in I
\end{array}
$$

The objective function C.9 maximizes the total probability that each constraint $i \in I$ is feasible. Alternatively, it can also be re-written to maximize the minimum value of $\alpha_{i}$ across all constraints. C.10 ensures that the solution's 
expected profit is within $\Delta$ units of the expected profit associated with the nom-

\section{Appendix D. $\Delta$ Model - General Formulation}

The standard binary integer program that is required to be made robust is:

$$
\begin{array}{ll}
\max & \sum_{j \in J} c_{j} x_{j} \\
\text { s.t. } & \sum_{j \in J} a_{i j} x_{j} \leq b_{i} \quad \forall i \in I \\
& x_{j} \in\{0,1\} \quad \forall j \in J,
\end{array}
$$

where the notation used is the same as that for C.1 - C.3. $a_{i j}$ for all $i \in I, j \in \bar{J}_{i}$, is subject to uncertainty, with $\tilde{a}_{i j}$ as its realized value. $a_{i j}$ is the nominal value of $\tilde{a}_{i j}$, and also the mean value of its symmetric range of variation. $\hat{a}_{i j}$ is the width of one half of the symmetric range of variation of $\tilde{a}_{i j}$, for all $i \in I, j \in J . \hat{a}_{i j}=0$ for $j \in J \backslash \bar{J}_{i} . x_{j}$ for all $j \in J$, is a binary decision variable that equals 1 if that variable is present in the solution and 0 otherwise. 
To avoid the need to specify $\Gamma$ values, Marla (2010) modifies the BertsimasSim (Bertsimas and Sim, 2004), (Bertsimas and Sim, 2003) formulation to inincreased robustness, the profit of a robust solution from the $\Delta$ formulation is at least $\sum_{j \in J} c_{j} y_{j}^{*}-\delta$. Let variables $v_{i j}$ equal 1 if the uncertain coefficient $\tilde{a}_{i j}$ is not allowed to take on its extreme value, and instead takes on its nominal value in the solution of the $\Delta$ model. Variables $w_{i l}$ equal 1 if there exists a $k \geq l$ with ${ }_{1350} \quad v_{i k}=1$, for $l=|J|-\left|\bar{J}_{i}\right|+1, \ldots,|J|+1 . w_{i l}$ variabless for $l=|J|-\left|\bar{J}_{i}\right|+1, \ldots,|J|+1$ in each constraint $i$ follow a non-increasing step function. 
This leads to the following $\Delta-E V$ formulation:

$$
\begin{aligned}
& \min \sum_{i \in I} \Delta_{i} \\
& \text { s.t. } \sum_{j \in J} c_{j} x_{j} \geq \sum_{j \in J} c_{j} y_{j}^{*}-\delta \\
& \sum_{j \in J}\left(a_{i j}+\hat{a}_{i j}\right) x_{j}-\sum_{j \in J} \hat{a}_{i j} v_{i j} \leq b_{i} \\
& \Delta_{i} \geq \sum_{l=|J|-\left|\bar{J}_{i}\right|+1}^{|J|}\left[\left(l-|J|+\left|\bar{J}_{i}\right|\right)\left(w_{i, l}-w_{i, l+1}\right)+v_{i, j(i, l)}-w_{i, l}\right] \quad \forall i \in I \\
& \Delta_{i} \geq \sum_{j \in J} v_{i j} \\
& \forall i \in I \quad(\mathrm{D} .8) \\
& v_{i j} \leq x_{j} \\
& \forall j \in J, \forall i \in I \\
& v_{i, j(i, l)} \leq w_{i, l} \\
& \forall l \in J \quad(\mathrm{D} .10) \\
& v_{i j} \geq x_{j}+w_{i, l(i, j)}-1 \\
& \forall j \in J, \forall i \in I \\
& w_{i, l+1} \leq w_{i, l} \\
& \forall l \in|J|-\left|\bar{J}_{i}\right|+1, \ldots,|J|, \forall i \in I \\
& w_{i,|J|-\left|J_{i}\right|+1} \geq 1 \\
& \forall i \in I \quad \text { (D.13) } \\
& w_{i,|J|+1}=0 \\
& \forall i \in I \quad \text { (D.14) } \\
& w_{i l}=0 \\
& \forall l \in 1, \ldots,|J|-\left|\bar{J}_{i}\right|, \forall i \in I \\
& \forall j \in J \\
& x_{j} \in\{0,1\} \\
& \forall j \in 1, \ldots,|J|, \forall i \in I \\
& v_{i j} \in[0,1] \\
& \forall l \in 1, \ldots,|J|+1, \forall i \in I
\end{aligned}
$$

The formulation is described as follows: The objective (D.4) is to minimize the sum of the number of coefficients in each constraint that must assume their nominal values to satisfy all constraints. This serves the purpose of trying to maximize the number of coefficients that can take their extreme values and still maintain feasibility. Constraints D.5 require that the profit from the 'robust' solution does not differ from the profit of the deterministic solution by more than a 'robustness budget' of $\delta$. Feasibility is assured by constraints 
(D.6). Variables $v_{i j}$ are set equal to 1 in constraint $i$, for all coefficients $j$ that worst-case values, under budget limitations.

\section{References}

Ageeva Y. Approaches to incorporating robustness into airline scheduling. Masters thesis, Massachusetts Institute of Technology; 2000.

1385

must be set to their nominal values. Inequalities (D.9) prevent a coefficient from taking on its nominal value unless the variable is present in the solution, that is, $v_{i j}$ is zero if $x_{j}$ is zero in the solution. Constraints (D.7) and (D.8) provide different mechanisms to count the maximum number of variables whose coefficients in constraint $i \in I$ are subject to uncertainty and must take on nominal values. The explanation for these constraints lies in the realization that when the columns are sorted in increasing order of $\hat{a}_{i j}$ values for each row $i$, the minimum number of coefficients that must assume nominal values to maintain feasibility is determined by forcing the smallest $\hat{a}_{i j}$ values (for which $\left.\hat{a}_{i j} \neq 0\right)$ in the solution to have their associated $v_{i j}$ values set to 1 , if variable are essentially the same, both are retained in order to provide better bounds on the solution. (D.10) requires the $w$ variable to be at least as large as the corresponding $v$ variable for that $j$ and $i$. Also, (D.11) forces $v$ to be 1 if both the corresponding $x$ and $w$ are 1. Constraints (D.12), (D.13) and (D.14) ensure that the $w_{i l}$ variables corresponding to uncertain coefficients in each $i$ form a step function. The $w_{i l}$ variables corresponding to coefficients that are not subject to uncertainty are zero D.15. The $x$ and $w$ variables are binary, as required by (D.16) and (D.18) respectively. We thus force the $v$ variables to take on binary values as well. One can think of this model as maximizing the total number of coefficients summed over all constraints that can take on their

AhmadBeygi S, Cohn A, Lapp M. Decreasing airline delay propagation by re-allocating scheduled slack. IIE transactions 2010;42:478-89. 
Air Traffic Association Annual Report. http://www.iata.

ㅇ org/NR/rdonlyres/84158349-7772-4892-86AB-836DE73E0A52/0/ IATAAnnualReport2008.pdf; 2008.

Arikan M, Deshpande V, Sohoni M. Building reliable air-travel infrastructure using empirical data and stochastic models of airline networks. Operations Research 2013;11:45-64.

Armacost A, Barnhart C, Ware K. Composite variable formulations for express shipment service network design. Transportation Science 2002;36:1-20.

Ball M, Barnhart C, Dresner M, Hansen M, Neels K, Odoni A, Peterson E, Sherry L, Trani A, Zou B. Total Delay Impact Study: A Comprehensive Assessment of the Costs and Impacts of Flight Delay in the United States. Technical Report; NEXTOR: The National Center of Excellence for Aviation Operations Research; 2010.

Barnhart C, Belobaba P, Odoni A. Applications of operations research in the air transport industry. Transportation Science 2003;37:368-91.

Ben-Israel A. On some problems of mathematical programming. Ph.D. thesis; Northwestern University; 1962.

Ben-Tal A, Nemirovski A. Robust convex optimization. Mathematical Operations Research 1998;23:769-805.

Ben-Tal A, Nemirovski A. Robust solutions to uncertain programs. Operations Research Letters 1999;25:1-13.

Ben-Tal A, Nemirovski A. Robust solutions of linear programming problems contaminated with uncertain data. Mathematical Programming 2000;88:41124.

Bertsimas D, Sim M. Robust discrete optimization and network flows. Mathematical Programming Series B 2003;98:49-71. 
Bertsimas D, Sim M. The price of robustness. Operations Research 2004;52(1):35-53.

1415

Bertsimas D, Thiele A. A robust optimization approach to supply chain management. Massachusetts Institute of Technology 2003;.

Bertsimas D, Thiele A. Robust and data-driven optimization: modern decision making under uncertainty; INFORMS. Tutorials on Operations Research. p. $195-22$.

${ }_{1420}$ Bureau of Transportation Statistics . Airline Service Quality Performance Database. http://aspm.faa.gov/asqp/sys//2009a.

Bureau of Transportation Statistics . On-time Performance - Flight Delays at a Glance. http://www.transtats.bts.gov/HomeDrillChart.asp 2009b.

Bureau of Transportation Statistics . http://www.transtats.bts.gov/

1425 a HomeDrillChart_Month.asp?Sel_Year=2016\&Arr_Del=1\&Sel_Carrier= 000\&Sel_Airport=000\&URL_SelectYear=\&URL_SelectMonth=, 2016.

Burke EK, De Causemaecker P, De Maere G, Mulder J, Paelinck M, Vanden Berghe G. A multi-objective approach for robust airline scheduling. Computers and Operations Research 2010;37:822-32.

Charnes A, Cooper WW. Chance constrained programming. Management Science 1959;6(1):73-9.

Charnes A, Cooper WW. Deterministic equivalents for optimizing and satisficing under chance constraints. Operations Research 1963;11(1):18-39.

Dunbar M, Froyland G, Wu C. Robust airline schedule planning: Minimizing propagated delay in an integrated routing and crewing framework. Transportation Science 2012;46:204-16.

Eggenberg N, Salani M, Bierlaire M. Ufo: Uncertainty feature optimization, an implicit paradigm for problems with noisy data. In: CTW. 2008 p. 72-7. 
Eggenberg N, Salani M, Bierlaire M. Uncertainty feature optimization: an implicit paradigm for problems with noisy data. Networks 2011;57(3):270-84. doi $10.1002 /$ net. 20428

Ehrgott M, Ryan D. Constructing robust crew schedules with bicriteria optimization. Journal of Multicriteria Decision Analysis 2002;11:139-50.

Froyland G, Maher SJ, Wu C. Recoverable robust single day aircraft maintenance routing problem. Computers and Operations Research 2014a;51:13045.

Froyland G, Maher SJ, Wu C. The recoverable robust tail assignment problem. Transportation Science 2014b;48:351-72.

Iancu DA, Trichakis N. Pareto efficiency in robust optimization. Management Science 2014;60.

Jiang H, Barnhart C. Dynamic airline scheduling. Transportation Science 2009;43:336-54.

Kang L, Clarke J. Degradable airline scheduling; 2002. Working paper, Operations Research Center, Massachusetts Institute of Technology.

Lan S, Clarke J, Barnhart C. Planning for robust airline operations: Optimizing aircraft routings and flight departure times to minimize passenger disruptions. Transportation Science 2006;40(1):15-28.

Marla L. Robust optimization for network-based resource allocation problems. Masters thesis, Massachusetts Institute of Technology; 2007.

Marla L. Airline Schedule Planning and Operations: Optimization-based Approaches for Delay Mitigation. Ph.D. thesis; Massachusetts Institute of Technology; 2010.

Marla L, Vaaben B, Barnhart C. Integrated disruption management and flight planning to trade off delays and fuel burn. Transportation Science 2016;Articles in Advance:1-24. URL: http://dx.doi.org/10.1287/trsc.2015.0609. 
Nemirovski A, Shapiro A. Convex approximations of chance-constrained programs. SIAM Journal of Optimization 2006;17:969-96.

Rosenberger JM, Johnson EL, Nemhauser GL. A robust fleet assignment model with hub isolation and short cycles. Transportation Science 2004;38:357-68.

Schaefer A, Johnson E, A. K, Nemhauser GL. Airline crew scheduling under uncertainty. Transportation Science 2005;39:340-8.

Shebalov S, Klabjan D. Robust airline crew pairing: Move-up crews; 2004. Working paper, the University of Illinois at Urbana-Champaign, Available from http://netfiles.uiuc.edu/klabjan/www.

Smith BC, Johnson E. Robust airline fleet assignment: Imposing station purity using station decomposition. Transportation Science 2006;40:497-516.

Sohoni M, Lee Y, Klabjan D. Robust airline scheduling under block time uncertainty. Transportation Science 2011;45:451-64.

Soyster AL. Convex programming with set-inclusive constraints and applications to inexact linear programming. Operations Research 1973;21.

US DoT . United States Department of Transportation website. http://www . dot.gov/new/index.htm 2009.

Weide O, Ryan D, Ehrgott M. An iterative approach to robust and integrated aircraft routing and crew scheduling. Computers and Operations Research 2010;37:833-44.

Yan C, Kung J. Robust aircraft routing. 2015. Transportation Research, forthcoming.

Yen J, Birge J. A stochastic programming approach to the airline crew scheduling problem. Transportation Science 2006;40:3-14. 